\title{
Nonparametric EWMA-Type Control Charts for Monitoring Industrial Processes: An Overview
}

\author{
Ioannis S. Triantafyllou \\ Department of Computer Science \& Biomedical Informatics, \\ University of Thessaly, Lamia 35131, Greece. \\ Corresponding author: itriantafyllou@uth.gr \\ Mangey Ram \\ Department of Mathematics, Computer Science \& Engineering, \\ Graphic Era Deemed to be University, Dehradun, Uttarakhand, India. \\ E-mail: drmrswami@geu.ac.in
}

(Received on February 2, 2021; Accepted on April 3, 2021)

\begin{abstract}
In the present paper we provide an up-to-date overview of nonparametric Exponentially Weighted Moving Average (EWMA) control charts. Due to their nonparametric nature, such memory-type schemes are proved to be very useful for monitoring industrial processes, where the output cannot match to a particular probability distribution. Several fundamental contributions on the topic are mentioned, while recent advances are also presented in some detail. In addition, some practical applications of the nonparametric EWMA-type control charts are highlighted, in order to emphasize their crucial role in the contemporary online statistical process control.
\end{abstract}

Keywords- Distribution-free statistical methods, Rank-based procedures, Nonparametric statistical process control, Exponentially weighted moving average (EWMA) control charts, Sign statistics.

\section{Introduction}

Statistical process control is implemented in industrial procedures and aims at improving their quality by monitoring the corresponding production process. Among others, monitoring schemes have been proved to be very useful statistical methods for supervising a measured characteristic of the product and tracking down plausible changes in the underlying distribution of the process. The most popular and easy-to-use monitoring schemes, which have been introduced and well-studied in the literature, are the so-called Shewhart-type, Cumulative Sum (CUSUM, hereafter) and Exponentially Weighted Moving Average (EWMA, hereafter) monitoring schemes. For a thorough consideration of the basic principles of the topic, the excellent monographs of Montgomery (2009) and Qiu. (2014) or the works of Woodall (2000) and Woodall and Montgomery (1999) are strongly recommended.

A broad variety of monitoring schemes have been already established under the presumption that the production data match adequately a specific probability distribution. Nevertheless, this demand is not often fulfilled and consequently it is evident that the distribution-based monitoring schemes do not constantly provide a reliable framework. To overcome this problem and at the same time maintain the common framework of a control chart, a broad variety of nonparametric schemes have been introduced in the literature. It is forthright that nonparametric (or distribution-free) control charts do not rest with the process distribution and therefore there is no need to assume one (or even worse to be obliged to know it). The general framework of the so-called Nonparametric 
International Journal of Mathematical, Engineering and Management Sciences

Vol. 6, No. 3, 708-751, 2021

https://doi.org/10.33889/IJMEMS.2021.6.3.044

Statistical Process Control and some fundamental perspectives of it could be found in the works of Chakraborti and Graham (2019a), Qiu and Li (2011a, 2011b) or Qiu (2018).

One of the classes of distribution-free schemes for supervising univariate processes, is the family of Shewhart-type nonparametric control charts (SNCC class, hereafter). The schemes, which are included in the $S N C C$ class, utilize an appropriate nonparametric test statistic, whose values are calculated by the aid of individual observations or random groups collected from the process. These observations are depicted and compared to the control limits of the corresponding chart, in order to draw a conclusion about the status of the production. The $S N C C$ class seems to include the easiestto-use control charts in comparison with the other families of distribution-free schemes. Some pioneer works on the $S N C C$ class are presented by the review paper of Chakraborti et al. (2001), while recent advances on the topic are discussed by Chakraborti and Graham (2019b) or Koutras and Triantafyllou (2020).

A second family of nonparametric schemes for monitoring univariate processes includes the socalled CUSUM nonparametric control charts (CNCC class, hereafter). Each member of this class calls for an appropriate nonparametric test statistic and afterwards the CUSUM procedure is constructed via the conventional approach. The $C N C C$ class outperforms the SNCC family for tracking down slight or middling changes of either the location or the scale parameter of the underlying process. For multivariate CUSUM nonparametric control charts, the works provided by Qiu and Hawkins (2001), Qiu (208) or Xue and Qiu (2020) are highly recommended.

The third family of nonparametric monitoring schemes contains memory-type statistical tools, known as nonparametric EWMA control charts (EWMANCC class, hereafter). These charts utilize an appropriate nonparametric testing procedure in order to build the corresponding $E W M A$ statistic, whose values are afterwards plotted and monitored by the aid of the respective control limits. It is evident that the EWMANCC class is supposed to be superior to the aforementioned SNCC class for tracking down small changes of the underlying distribution process. Throughout the lines of the present manuscript, the EWMANCC class is under investigation. Two main subgroups of the EWMANCC class are studied in detail. The first one includes the EWMA-type schemes based on sign statistics, while the second subgroup contains the corresponding rank-based ones. Our study is devoted only to these subgroups due to their enormous growth during the last decade. It is noticeable that over 20 different nonparametric control charts, which belong to the aforementioned subgroups, have been introduced in the last two years. However, it is important to mention that a variety of EWMA-type nonparametric monitoring schemes based on alternative approaches have been also established in the literature (see, e.g. Zou and Tsung, 2010; Graham et al., 2012, 2016;) Qiu et al., 2018; You and Qiu, 2020).

The review article is worked out as follows: Section 2 offers some general notations and the framework for constructing an EWMA control chart. A review of univariate nonparametric EWMAtype control schemes which rely on sign statistics is discussed in Section 3. Section 4 offers a detailed presentation of EWMA -type rank-based monitoring schemes, while Section 5 puts forth the general outline of the present manuscript, whilst some hints for further research on the topic are also dropped.

\section{General Notations and EWMA Framework}

In the present section, general notations are presented in order to make the remaining manuscript more readable. The main symbols which are utilized in the next sections, are listed below. However, 
International Journal of Mathematical, Engineering and Management Sciences

Vol. 6, No. 3, 708-751, 2021

https://doi.org/10.33889/IJMEMS.2021.6.3.044

some specific notations which are not generally used by all charts, are properly introduced and defined throughout the lines of the corresponding subsection of the present review article.

$E(X)$ : the expected value of random variable $X$.

ARL: Average Run Length.

$\operatorname{Var}(X)$ : the variance of random variable $X$.

PCI: Performance Comparison Index.

sign (a): the sign of number $a$.

MRL: Median Run Length.

SR: Wilcoxon signed-rank statistic.

$I(b)$ : an indicator (dummy) variable depending on $b$.

RARL: Relative Average Run Length.

LCL: Lower Control Limit.

EQL: Extra Quadratic Loss.

SDRL: Standard Deviation of Run Length.

UCL: Upper Control Limit.

Generally speaking, the EWMA control charts provide an attractive alternative monitoring tool, when small changes in the location or scale parameter are investigated. In fact, a EWMA scheme seems to be quite capable for Phase II process monitoring cases. The behavior of the EWMA schemes is roughly similar to that of the Cumulative Sum control charts but it seems to be user friendly (see, e.g. Roberts, 1959; Montgomery, 2009).

Due to the fact that EWMA charts mix together knowledge from different samples, they are proved to be more sensitive than Shewhart-type schemes for tracking down slight changes. If a change in the process is under way, the EWMA chart is expected to produce a signal, while an adjustment should take place for reinstating the process back on the desirable performance level. A graphical representation of the general framework for constructing a EWMA chart follows the ordinary scheme, namely the weighted average statistic of all historical and present data is plotted versus the identification number and the conclusion about the status of the process is drawn by comparing its observed values to the corresponding control limits (see, e.g. Montgomery, 2009). It is worth mentioning that after the EWMA scheme has been implemented for plenty time sessions, the control limits are expected to gain on steady-state values. A graphical representation of the general framework for constructing a EWMA chart follows the ordinary scheme, namely the exponentially weighted average statistic is plotted against the identification number and the conclusion if the process can be characterized as in- or out-of-control can be drawn by comparing its observed values to the corresponding control limits (see, e.g. Montgomery, 2009).

\section{Nonparametric EWMA Schemes Based on Sign Statistics}

In the present section, univariate nonparametric control schemes, which rely on sign statistics and follow the general EWMA framework are discussed. It is evident that the sign statistic and its modifications have been already implemented for building up Shewhart-type distribution-free schemes (see, e.g. Asghari et al., 2018; Castagliola et al., 2020). However, our study deals with the corresponding EWMA-type advances. 
International Journal of Mathematical, Engineering and Management Sciences

Vol. 6, No. 3, 708-751, 2021

https://doi.org/10.33889/IJMEMS.2021.6.3.044

Generally speaking, the EWMA framework relies on an exponentially weighted moving average of the ongoing and historical data. In simple words, if $Y_{t}, t=1,2, \ldots$ denotes the sequentially observed data, then the monitoring EWMA-type statistic $Z_{t}$ can be expressed as

$Z_{t}=\lambda Y_{t}+(1-\lambda) Z_{t-1}, t=1,2, \ldots$

where the starting value is often determined to be equal to the corresponding target value $\mu_{0}$ of the underlying process (see, e.g. Lucas and Saccucci, 1990). Note that the control limits of the resulting scheme are defined via the standard deviation $\sigma_{Z}$ of the EWMA-type statistic appeared in (1) and are usually chosen as $\mu_{0} \pm L \sigma_{Z}$, where the design parameter $L$ is appropriately determined such that the desired performance level is reached. For some fundamental advances on the topic of EWMA-type control charts, the works provided by Hunter (1986) or Roberts (1959) are recommended.

When a process is under investigation, a common way to monitor its performance is to utilize the so-called sign statistic for providing some evidence about the behavior of a specific measurable characteristic of the product. If we denote by $\tau$ the target value of the underlying quality characteristic, the sign statistic is simply an indicator variable, which provides the information whether each observed value of the underlying characteristic is above or below the target value. However, plenty alterations of the traditional sign statistic have been already studied and the corresponding control charts have been studied in detail.

We next provide a detailed review of nonparametric univariate monitoring schemes based on sign statistics, namely of these members of the EWMANCC class which employ the well-known sign statistic or its modifications. The set-up of presentation of the included articles is determined to be chronological for the purpose of setting up a comprehensible and well-structured flow.

\subsection{Nonparametric EWMA Control Charts Based on Wilcoxon Signed-Rank Statistic} Amin and Searcy (1991) established a distribution-free EWMA control chart based on the Wilcoxon signed-rank statistic (see, e.g. Wilcoxon, 1945). Their scheme seems to be proper for supervising a production when the underlying distribution is unascertained or there is lack of knowledge about its variance or shape. Based on a simulation study, Amin and Searcy (1991) confirmed the superiority of their nonparametric scheme against the traditional $\bar{X}-E W M A$ scheme under heavy-tailed distributions.

Let $X_{t 1}, X_{t 2}, \ldots, X_{t g}, t=1,2, \ldots$ denote the observations of the $t$-th sample of magnitude $g$ drawn independently and sequentially from the process. If $R_{t j}$ corresponds to the rank of the $j$-th observation in the sample consisting of the respective absolute values, namely $\left|X_{t 1}\right|,\left|X_{t 2}\right|, \ldots,\left|X_{t g}\right|, t=1,2, \ldots$, then the so-called Wilcoxon signed-rank statistic is defined as

$U_{t j}=\operatorname{sign}\left(X_{t j}\right) R_{t j}, j=1,2, \ldots, g$ 
International Journal of Mathematical, Engineering and Management Sciences

Vol. 6, No. 3, 708-751, 2021

https://doi.org/10.33889/IJMEMS.2021.6.3.044

where $\operatorname{sign}(a)$ takes on the value $1(-1)$ if the quantity $a$ is positive (negative). Amin and Searcy (1991) considered the following EWMA-type monitoring statistic

$Z_{t}=(1-\lambda) Z_{t-1}+\lambda S R_{t}, 0<\lambda<1$

where $S R_{t}=\sum_{j=1}^{g} U_{t j}$, denotes the signed-rank statistic for the $t$-th random sample of magnitude $g$.

The proposed two-sided EWMA-type scheme generates an out-of-control indication if the monitoring statistic $Z_{t}$ violates the upper or the lower control limit. It should be mentioned that in order for the corresponding chart to be capable to generate an alarm due to an observed random sample, the maximum value for the corresponding upper control limit is determined as $\lambda g(g+1) / 2$ which actually refers to maximum value that may be calculated for the variable $Z_{t}$ given that $Z_{0}$ equals to zero.

Amin and Searcy (1991) also investigated how the presence of autocorrelation between the observed values affects the behavior of the resulting scheme. Considering the first order autoregressive time series model of Box and Jenkins (1976), Amin and Searcy (1991) concluded that the in-control $A R L$ of the respective scheme change roughly in a linear fashion as the autoregressive coefficient of the aforementioned model changes, in contrast with the exponential change of the corresponding $A R L$ of the common EWMA procedure. For a thorough discussion about the above-mentioned issue, the interested reader is referred to Hunter (1990), MacGregor and Harris (1990) or Baxley (1990).

As Amin and Searcy (1991) argued, the proposed nonparametric EWMA monitoring scheme seems to be affected slightly by the specific choice of the corresponding weighting parameter. Amin and Searcy carried out a numerical experimentation based on different distributional models, such as the Normal distribution, the Uniform distribution, the Double Exponential distribution, the Gamma distribution and the Cauchy distribution. According to their simulation results, the proposed nonparametric EWMA monitoring scheme performs satisfactorily for non-normal or heavy-tailed distributions.

In addition, Graham et al. (2011a) studied the nonparametric EWMA control chart established by Amin and Searcy (1991) in some detail. Implementing a suitable Markov chain technique, the above-mentioned authors determined the distribution of the run length for the resulting scheme given that the process has not shifted, while practical guidance and suggestions for picking up its design parameters are also documented. Based on several quantitative measurements, just like the common $A R L$, the $M R L$ or the $S D R L$, authors appraised the in- and out-of-control attribution of the nonparametric EWMA control scheme established by Amin and Searcy (1991). Their simulation procedure relied on random samples generated by several distributions, such as Standard Normal, scaled Student's t, Laplace or contaminated Normal distribution.

The control limits of the above-mentioned chart are given as

$$
L C L=-L \sqrt{\frac{g(g+1)(2 g+1)}{6} \frac{\lambda}{2-\lambda}}, \quad U C L=L \sqrt{\frac{n(n+1)(2 n+1)}{6} \frac{\lambda}{2-\lambda}}
$$


Furthermore, Abid et al. (2017) developed a nonparametric EWMA signed-rank control chart using the so-called ranked set sampling (see, e.g. McIntyre (1952)). More specifically, they utilized the Wilcoxon signed-rank statistic defined earlier and proposed a new edition of the EWMA signedrank monitoring scheme by implementing ranked set sampling. For some recent advances on the ranked set sampling procedure, the interested reader is referred to Koshti and Kamalja (2021) or Frey and Zhang (2021), while some comprehensive details about the above-mentioned sampling scheme are provided by Hettmansperger (1995), Kvam and Samaniego (1994) or Dell and Clutter (1972). Their proposed chart seems to be more capable for tracking down plausible changes in the underlying distribution, when it is compared to other existing nonparametric EWMA schemes.

Let us presume that a group of magnitude $g$ is drawn randomly from the underlying process for supervising the process location $\mu_{0}$. Since Abid et al. (2017) implemented the ranked set sampling with $m$ cycles, the proposed testing procedure is based on the quantity $R_{(l k) j}^{+}$, which corresponds to the within-group absolute rank of deviations from the target value $\mu_{0}$, e.g. $R_{(l k) j}^{+}=\left|X_{(l k) j}-\mu_{0}\right|$, $l=1,2, \ldots$ and $k=1,2, \ldots, g$. The nonparametric signed rank statistic under the ranked set sampling framework is given by (see, e.g. Kim and Kim (1996)).

$$
S R_{R S S_{i}}=\sum_{k=1}^{g} \sum_{j=1}^{m} \operatorname{sign}\left(X_{(l k) j}-\mu_{0}\right) R_{(p h) i}^{+}, l=1,2, \ldots, k=1,2, \ldots, g \text { and } j=1,2, \ldots, m
$$

while

$$
\operatorname{sign}\left(X_{(l k) j}-\mu_{0}\right)= \begin{cases}1, & \text { if } X_{(l k) j}-\mu_{0}>0 \\ 0, & \text { if } X_{(l k) j}-\mu_{0}=0 \\ -1, & \text { if } X_{(l k) j}-\mu_{0}<0 .\end{cases}
$$

The in-control expected value and the variance of the test statistic defined in Eq. (5) are given by

$$
E\left(S R_{R S S}\right)=0, \operatorname{Var}\left(S R_{R S S}\right)=(g m(g m+1)(2 g m+1) / 6) \delta_{0}^{2}
$$

where

$$
\delta_{0}^{2}=1-(4 / n) \sum_{k=1}^{g}\left(F_{k}(0)-1 / 2\right)^{2}
$$

while $F_{k}(0)$ can be computed by the aid of the incomplete beta integral, e.g.

$$
F_{k}(0)=\frac{(g m) !}{(k-1) !(g m-k) !} \int_{-\infty}^{0} F^{k-1}(u)(1-F(u))^{g m-k} f(u) d u .
$$


International Journal of Mathematical, Engineering and Management Sciences

Vol. 6, No. 3, 708-751, 2021

https://doi.org/10.33889/IJMEMS.2021.6.3.044

Abid et al. (2017) proposed a distribution-free EWMA scheme, which takes into account the following plotted statistic:

$$
E W M A_{S R_{R S S_{i}}}=\lambda S R_{R S S_{i}}+(1-\lambda) E W M A_{S R_{R S S_{i-1}}}
$$

where $S R_{R S S_{i}}$ is defined in Eq. (5) and $\lambda$ is the smoothing parameter of the proposed EWMA scheme $(0<\lambda \leq 1)$. The abovementioned statistic is plotted against the control limits:

$L C L_{S R_{R S S_{i}}}=-L \sqrt{\frac{g m(g m+1)(2 g m+1)}{6} \frac{\lambda}{2-\lambda} \delta_{0}^{2}}, U C L_{S R_{R S S_{i}}}=L \sqrt{\frac{g m(g m+1)(2 g m+1)}{6} \frac{\lambda}{2-\lambda} \delta_{0}^{2}}$

and the proposed distribution-free scheme generates an out-of-control indication whenever $E W M A_{S R_{R S S_{i}}} \geq U C L_{S R_{R S S_{i}}}$ or $E W M A_{S R_{R S S_{i}}} \leq L C L_{S R_{R S S_{i}}}$. It is forthright that the design parameter $L$ of the resulting chart is appropriately determined so that a required degree of attribution is achieved.

On the other hand, Perdikis et al. (2020b) studied a altered distribution-free EWMA-type control scheme which utilizes the Wilcoxon signed rank statistic. In order to determine both the in- and out-of-control $A R L$ of the resulting chart, Perdikis et al. (2020b) utilized a continuous transformation of the Wilcoxon signed rank statistic, combined with the common Markov chain technique (see, e.g. Brook and Evans, 1972). In their framework, the structure of an EWMA scheme which could be proved sensitive in tracking down increases in the quality measurement of interest is only considered.

Based on Eq. (2), one may readily deduce that the so-called Wilcoxon signed-rank statistic coincides to the sum of the signed ranks defined on $\{-g(g+1) / 2,-g(g+1) / 2+2, \ldots, g(g+1) / 2-2, g(g+1) / 2\}$. Consequently, the Wilcoxon signed-rank statistic $S R_{t}$ can be rewritten as:

$S R_{t}=2 S R_{t}^{+}-\frac{g(g+1)}{2}$

where $S R_{t}^{+}$corresponds to the sum of the positive ranks. Note that following a generating function approach proposed by McCornack (1965), the probability mass function of variable $S R_{t}^{+}$could be readily delivered. Implementing a parallel argumentation as the one proposed by Wu et al. (2020), Perdikis et al. (2020b) suggested to transform the statistic $S R_{t}$ into a new continuous one denoted by $S R_{t}^{*}$, whose probability density function can be expressed by the aid of the corresponding function of variable $S R_{t}^{+}$. Consequently, the monitoring statistic of the proposed continuousified one-sided EWMA-type control chart is given by:

$Z_{t}=\max \left(0, \lambda S R_{t}^{*}+(1-\lambda) Z_{t-1}^{*}\right)$, with $Z_{0}^{*}=0$ 
International Journal of Mathematical, Engineering and Management Sciences

Vol. 6, No. 3, 708-751, 2021

https://doi.org/10.33889/IJMEMS.2021.6.3.044

where $\lambda$ corresponds to the smoothing parameter. The upper-sided EWMA-type monitoring scheme proposed by Perdikis et al. (2020b) generates an out-of-control alarm if the statistic defined in Eq. (12) exceeds the corresponding upper control limit, which is given as:

$U C L=E\left(S R_{t}^{*}\right)+k \sqrt{\frac{\lambda}{2-\lambda} V\left(S R_{t}^{*}\right)}$

where

$E\left(S R_{t}^{*}\right)=\frac{g(g+1)\left(2 p_{0}-1\right)}{2}, V\left(S R_{t}^{*}\right)=\frac{2 g(g+1)(2 g+1) p_{0}\left(1-p_{0}\right)}{3}+\sigma^{2}$,

while $p_{0}$ corresponds to the in-control probability of recording an observation greater than the target value and $\sigma$ denotes the "continuousify" parameter. Perdikis et al. (2020b) investigated the efficiency of their proposal by utilizing plenty kernels apart from the Gaussian and they deduced that no important differences are present. Note that their study was mostly aimed at offering a powerful approach for examining the chart's run length attributes rather than confirming its supremacy against other competitors.

In addition, Perdikis et al. (2020a) established a nonparametric EWMA-type control chart based on the recursive formula introduced by Rakitzis et al. (2015), where the Wilcoxon signed rank statistic defined earlier is now utilized. An appropriate discrete Markov chain technique is implemented for determining the $A R L$ of both in- and out-of-control cases. Perdikis et al. (2020a) considered the monitoring statistic $Y_{t}$ which is obtained by the aid of the following formula:

$\left(\gamma_{t}+\gamma_{y}\right) Y_{t}+R_{t}=\gamma_{x} S R_{t}+\gamma_{y} Y_{t-1}+R_{t-1}$

where $\gamma_{t}, \gamma_{y}$ are two fixed positive integer-valued parameters, the initial values $R_{0}$ and $Y_{0}$ are equal to zero and $S R_{t}$ is given by Eq. (11).

Given that the distribution of test statistic $S R_{t}$ under the hypothesis that the process has not shifted, is proved to be symmetric around 0 , the corresponding limits and the center line of the proposed scheme coincide to $L C L=-K, C L=0$ and $U C L=K$ respectively, where $K$ takes on values from the set $\{2,4, \ldots, g(g+1) / 2\}$. It is straightforward that the proposed nonparametric EWMA scheme proposed by Perdikis et al. (2020a) produces an out-of-control signal if $Y_{t} \geq K$ or $Y_{t} \leq-K$. In order to optimize the performance of their proposed monitoring scheme, Perdikis et al. (2020a) utilized an appropriate algorithmic procedure for finding out the optimal combination of the design parameters, which results in the minimum respective out-of-control $A R L$.

\subsection{Nonparametric EWMA Sign Schemes Based on Sequential Records}

Yang et al. (2011) established a nonparametric EWMA control scheme for variables data to supervise the dispersion from the process target, without presuming a specific distributional model. Based on independent random samples of magnitude $n$, say $X_{1}, X_{2}, \ldots, X_{n}$, which are drawn from the process, the variables $Y_{j}$ and $I_{j}$ are defined as: 
International Journal of Mathematical, Engineering and Management Sciences

Vol. 6, No. 3, 708-751, 2021

https://doi.org/10.33889/IJMEMS.2021.6.3.044

$Y_{j}=X_{j}-T$ and $I\left(Y_{j}>0\right)=\left\{\begin{array}{l}1, \text { if } Y_{j}>0 \\ 0, \text { otherwise }\end{array}, j=1,2, \ldots, n\right.$

respectively. If $M$ corresponds to the total number of positive $Y_{j}$ 's, then it is straightforward that the random sum $M=\sum_{j=1}^{n} I\left(Y_{j}>0\right)$, follows the Binomial distribution with parameters $n$ and $p=0.5$, under the presumption that the process is in-control. Given that, instead of dispersion, one may supervise the corresponding process proportion $p$, Yang et al. (2011) established a distribution-free EWMA scheme, where the following monitoring statistic is plotted.

$$
E W M A_{M_{i}}=\lambda M_{i}+(1-\lambda) E W M A_{M_{i-1}}, 0<\lambda \leq 1
$$

where $M_{i}$ corresponds to the i-th sequentially recorded value of variable $Y_{i}$. Assuming that the starting value of the EWMA chart equals to the mean of $M$, namely $E\left(E W M A_{M_{i}}\right)=n / 2$, the corresponding variance can be expressed as $\operatorname{Var}\left(E W M A_{M_{i}}\right)=\frac{\lambda}{2-\lambda} \frac{n}{4}$. The control limits of the nonparametric EWMA sign monitoring scheme of Yang et al. (2011) are given below:

$$
L C L_{E W M A_{M}}=n / 2-L \sqrt{\frac{n}{4} \frac{\lambda}{2-\lambda}}, U C L_{E W M A_{M}}=n / 2+L \sqrt{\frac{n}{4} \frac{\lambda}{2-\lambda}}
$$

It goes without saying that the resulting EWMA scheme generates an alarm whenever $E W M A_{M_{i}} \geq U C L_{M_{i}}$ or $E W M A_{M_{i}} \leq L C L_{M_{i}}$. Note that the design parameters are determined appropriately for accomplishing a pre-specified performance level. Note that Yang et al. (2011) also applied the so-called arcsine transformation (see, e.g. Mosteller and Youtz, 1961) and constructed a revised version of their proposed control chart. A discussion referring to the abovementioned work is provided by Abbasi (2012).

Following their proposal, Aslam et al. (2014) modified the distribution-free EWMA scheme which relies on the sign statistic defined earlier, by implementing the so-called repetitive sampling procedure. Generally speaking, the aforementioned sampling scheme, which has been established by Sherman (1965), seems to be easy-to-use and more capable than the corresponding single or double sampling procedures (see, e.g. Balamurali and Jun, 2006).

Since the repetitive sampling scheme is activated, the conclusion about the status of the process relies on a single sample only if it is conspicuous, but resampling will be considered if it is questionable. In other words, the nonparametric EWMA monitoring scheme proposed by Aslam et al. (2014) is constructed by the aid of double control limits, namely two different lower and two separate upper control limits are calculated and considered simultaneously for monitoring the underlying process. More precisely, the distribution-free control chart considered by Aslam et al. (2014) generates an alarm whenever one of the following occurs: 


$$
E W M A_{M_{i}} \geq U C L_{1} \text { or } E W M A_{M_{i}} \leq L C L_{1}
$$

where

$$
L C L_{1}=n / 2-k_{1} \sqrt{\frac{n}{4} \frac{\lambda}{2-\lambda}}, U C L_{1}=n / 2+k_{1} \sqrt{\frac{n}{4} \frac{\lambda}{2-\lambda}}
$$

On the other hand, if

$$
L C L_{2} \leq E W M A_{M_{i}} \leq U C L_{2}
$$

where

$$
L C L_{2}=n / 2-k_{2} \sqrt{\frac{n}{4} \frac{\lambda}{2-\lambda}}, U C L_{2}=n / 2+k_{2} \sqrt{\frac{n}{4} \frac{\lambda}{2-\lambda}}
$$

then the process is characterized as in-control. Of course, the case where the monitoring statistic is located between the two upper control limits $U C L_{1}, U C L_{2}$ or between the two lower control limits $L C L_{1}, L C L_{2}$, is not covered by the above-mentioned conditions stated in (19) and (21). In such case, the proposed procedure calls for resampling, namely a different random sample of the same magnitude is collected from the process and the EWMA sign statistic is computed once again by the aid of formula given above. For illustration purposes, Aslam et al. (2014) applied the proposed EWMA nonparametric control chart for real data concerning the fill volume of soft-drink beverage bottles (see, e.g. Montgomery, 2009).

Moreover, Abid et al. (2016) introduced a distribution-free EWMA scheme which utilizes the sign test statistic and implements the so-called ranked set sampling, which seems to be useful especially when measurements are ruinous or costly. The proposed distribution-free scheme is an attractive second choice instead of the common distribution-free sign scheme mentioned earlier. Under the ranked set sampling, a single group of magnitude $n$ is collected randomly from a particular pool and afterwards, authorities order the units. The observation with the lowest rank is detected and forwarded for quantification. The rest $n-1$ units are put back to the pool and a different group of same size is collected. Again, the authorities rank the units and the second lowest is detected, quantified and the rest $n-1$ units are put back once again. The process is done over until $n$ ordered units are measured, say $X_{(1) 1}, X_{(2) 1}, \ldots, X_{(n) 1}$, which corresponds to the random sample of the first cycle. The same procedure is realized for $m$ repetitions and an ordered group of magnitude $r=n m$ is obtained. Some advances on ranked-set-sampling-based process control, are provided by Abujiya and Muttlak (2004), Muttlak and Al-Sabah (2003), Al-Omari and Haq (2012), Mehmood et al. (2013) or Haq et al. (2015).

The most handy conformation of the ranked set sampling sign test statistic can be viewed as (see, e.g. Hettmansperger, 1995). 
$S R_{R S S}^{+}=\sum_{j=i}^{n} \sum_{i=1}^{m} I\left(X_{(j) i}-\mu_{0}>0\right)$,

where the above-mentioned variable $I()$ can be expressed as:

$I\left(X_{(j) i}-\mu_{0}\right)= \begin{cases}1, & \text { if } X_{(j) i}-\mu_{0}>0 \\ 0, & \text { otherwise }\end{cases}$

Note that the test statistic defined in Eq. (18) can be viewed as a sum of $n S_{(j)}^{+}, j=1,2, \ldots, n$, where $S_{(j)}^{+}=\sum_{i=1}^{m} I\left(X_{(j) i}-\mu_{0}>0\right)$, follows a Binomial distribution with parameters $m$ and $1-H_{j}(0)$. Consequently, the expected value and the variance of the test statistic defined in Eq. (23) are given by:

$$
E\left(S R_{R S S}^{+}\right)=n m / 2, \operatorname{Var}\left(S R_{R S S}^{+}\right)=(n m / 4) \delta_{0}^{2}
$$

where $\delta_{0}^{2}$ is defined earlier.

Abid et al. (2016) proposed a distribution-free EWMA scheme, which plots the following random variable:

$$
E W M A_{S R_{R S S_{i}}^{+}}=\lambda S R_{R S S_{i}^{+}}+(1-\lambda) E W M A_{S R_{R S S_{i-1}}^{+}}
$$

where $S R_{R S S_{i}}^{+}$is defined in Eq. (23) and $\lambda$ is the smoothing parameter of the proposed EWMA chart $(0<\lambda \leq 1)$. The abovementioned statistic is plotted against the control limits:

$$
L C L_{S R_{R S S_{i}^{+}}}=m n / 2-k \sqrt{\frac{m n}{4} \frac{\lambda}{2-\lambda} \delta_{0}^{2}}, U C L_{S R_{R S S_{i}^{+}}}=m n / 2+k \sqrt{\frac{m n}{4} \frac{\lambda}{2-\lambda} \delta_{0}^{2}}
$$

and the proposed distribution-free scheme generates an alarm if $E W M A_{S R_{R S S_{i}^{+}}} \geq U C L_{S R_{R S S_{i}^{+}}}$or $E W M A_{S R_{R S S_{i}^{+}}} \leq L C L_{S R_{R S S_{i}^{+}}}$. It goes without saying that the design parameter $k$ of the resulting chart corresponds to the distance between the lower and upper limit and is appropriately determined for reaching a desired level of attribution.

Castagliola et al. (2019) established a distribution-free EWMA-type sign scheme for supervising plausible changes in the location parameter of the underlying process. Implementing the approach introduced by Raktizis, Castagliola and Maravelakis (2015), Castagliola et al. (2019) considered the monitoring statistic $Y_{t}$ which is obtained by the aid of the following formula:

$$
\left(\gamma_{t}+\gamma_{y}\right) Y_{t}+R_{t}=\gamma_{x} S N_{t}+\gamma_{y} Y_{t-1}+R_{t-1}
$$


where $\gamma_{t}, \gamma_{y}$ are two fixed positive integer-valued parameters, $S N_{t}=\sum_{j=1}^{n} \operatorname{sign}\left(X_{(t) j}-\mu_{0}\right)$, and

$$
\operatorname{sign}\left(X_{(t) j}-\mu_{0}\right)=\left\{\begin{array}{l}
1, \text { if } X_{(t) j}-\mu_{0}>0 \\
0, \text { if } X_{(t) j}-\mu_{0}=0 \\
-1, \text { if } X_{(t) j}-\mu_{0}<0 .
\end{array}\right.
$$

Given that the distribution of test statistic $S N_{t}$ under the hypothesis that the process has not shifted, is symmetric around 0 , the control limits and the center line of the proposed scheme are equal to $L C L=-K, C L=0$ and $U C L=K$ respectively, where $K$ takes on integer even values equal to or greater than 2 and less than or equal to the even $n$, or integer odd values greater than or equal to 1 and less than or equal to the odd $n$. It is straightforward that the proposed distribution-free EWMA scheme proposed by Castagliola et al. (2019) generates an alarm if $Y_{t} \geq K$ or $Y_{t} \leq-K$.

\subsection{A Distribution-Free Sign Scheme Based on Individual Measurements}

Graham et al. (2011b) established a distribution-free EWMA sign scheme for independent and identically distributed individual observations. A Markov chain technique is implemented for deducing the distribution of run length for the resulting scheme, while several performance characteristics are studied in order to shed light on its in- and out-of-control behavior. Graham et al. (2011b) considered the well-known sign statistic $S N_{i}$ defined as:

$S N_{i}=\operatorname{sign}\left(X_{i}-\theta_{0}\right), i=1,2, \ldots$

where $\theta_{0}$ corresponds to the target value. In their framework, the monitoring statistic $Z_{i}$ of the proposed EWMA scheme is deduced by successively accumulating the sign statistics $S N_{i}, i=1,2, \ldots$ as:

$$
Z_{i}=\lambda S N_{i}+(1-\lambda) Z_{i-1}, Z_{0}=0
$$

whereas $\lambda$ denotes its smoothing parameter $(0<\lambda \leq 1)$. Note that Graham et al. (2011b) considered only the median value among other available percentiles and consequently throughout the lines of their study, the parameter $\theta_{0}$ expresses the in-control process median. Following their choice, the control limits of the proposed EWMA nonparametric monitoring scheme are given by:

$$
L C L=-L \sqrt{\frac{\lambda}{2-\lambda}}, U C L=L \sqrt{\frac{\lambda}{2-\lambda}}
$$

Graham et al. (2011b) presented several combinations of the design parameters $\lambda$ and $L$, which result in a required degree of in-control attribution. Based on the above-mentioned results, the practitioner has at his/her disposal a useful guidance for implementing the proposed control chart. 
International Journal of Mathematical, Engineering and Management Sciences

Vol. 6, No. 3, 708-751, 2021

https://doi.org/10.33889/IJMEMS.2021.6.3.044

Graham et al. (2011b) appraised the performance of the proposed EWMA monitoring scheme by considering different distributional models. Their numerical experimentation included symmetric and asymmetric distributions, light- and heavy-tailed distributions, while the effect of outliers was also studied. For slighter changes, the common EWMA scheme tracks down changes faster than the nonparametric one if the normal distribution is assumed. Conversely, the proposed nonparametric monitoring scheme is more capable versus the ordinary EWMA scheme under the more highly asymmetric and the more heavy-tailed distributions, such as the Laplace or Gamma case.

\subsection{An Extended Distribution-Free EWMA Sign Scheme}

Lu (2015) established a nonparametric generally weighted moving average (GWMA) sign scheme, which aims at improving the detection ability in slight changes of the process. The proposed scheme adopts the framework of the distribution-free scheme introduced by Yang et al. (2011) and extends the idea to the so-called generally weighted moving average scheme (see, e.g. Sheu and Lin, 2003).

Let $X$ be the measurement of concern with target value $\theta$, while $Y=X$ - $\theta$ expresses the corresponding distance of $X$ from the target. In this framework, Lu (2015) defined the process proportion $p$ as the probability of observing positive $Y$ 's and consequently the process is declared to be in-control (out-of-control) if $p$ equals to (is unequal with) 0.5 . Based on independent random samples of magnitude $n$, say $X_{1 t}, X_{2 t}, \ldots, X_{n t}, t=1,2, \ldots$, which are drawn from the process at time $t$, the variables $Y_{i t}$ and $I$ are defined as:

$Y_{i t}=X_{i t}-\theta$ and $I\left(Y_{i t}>0\right)=\left\{\begin{array}{l}1, \text { if } Y_{i t}>0 \\ 0, \text { otherwise }\end{array}\right.$

respectively. If $M_{t}$ corresponds to the total number of positive deviations $Y_{i t}{ }^{\prime} \mathrm{s}$, then it is straightforward that the random sum $M_{t}=\sum_{i=1}^{n} I\left(Y_{i t}>0\right)$, follows the Binomial distribution with parameters $n$ and $p=0.5$, under the presumption that the process is in-control. $\mathrm{Lu}$ (2015) introduced the nonparametric GWMA monitoring scheme, whose plotted statistic is given by:

$$
G_{t}=\sum_{j=1}^{t}\left(w^{(j-1)^{a}}-w^{j^{a}}\right) M_{t-j+1}+w^{t^{a}} \theta, 0 \leq w<1,0<a \leq 1
$$

where $M_{t}$ corresponds to the observation from the production at time $t$. Assuming that the starting value of the aforementioned scheme equals to the mean of $M_{t}$, namely $G_{0}=n / 2$, the corresponding variance can be expressed as $\operatorname{Var}\left(G_{t}\right)=B \frac{n}{4}$, where $B=\lim _{t \rightarrow \infty} \sum_{j=1}^{t}\left(\left(w^{(j-1)^{a}}-w^{j^{a}}\right)^{2}\right)$

The control limits of the nonparametric GWMA sign monitoring scheme of Lu (2015) are given below: 
International Journal of Mathematical, Engineering and Management Sciences

Vol. 6, No. 3, 708-751, 2021

https://doi.org/10.33889/IJMEMS.2021.6.3.044

$L C L=n / 2-L \sqrt{\frac{n}{4} B}, U C L=n / 2+L \sqrt{\frac{n}{4} B}$

It goes without saying that the resulting nonparametric sign chart generates an alarm whenever $G_{t} \geq U C L$ or $G_{t} \leq L C L$. Note that the design parameters are determined appropriately in order to reach the pre-determined degree of attribution. It is noteworthy that the nonparametric chart proposed by $\mathrm{Lu}(2015)$ is a generalization of the respective EWMA monitoring scheme proposed by Yang et al. (2011). Indeed, if we consider the special case $w=1-\lambda$, then Eq. (34) reduces to the sign control chart of the above-mentioned authors. Due to some simulation results, Lu (2015) concluded that the proposed GWMA monitoring scheme is proved to be more efficient for tracking down small process changes versus other EWMA schemes considered, while its implementation is strongly recommended under large values of the parameter $w$ and $a$.

\subsection{A Distribution-Free Sensitive EWMA Sign Scheme on A Moving Pattern}

Riaz (2015) proposed a distribution-free scheme which relies on moving sign statistics in an EWMA framework. Let $X$ be the measurement of our concern with target value $\mu$, while $Y=X-\mu$ expresses the corresponding distance of $X$ from the objective. In a similar framework as the one applied by Lu (2015), the probability $p$ of observing positive $Y$ 's could be utilized for monitoring the process location parameter. For an in-control process, probability $p$ is presumed to be equal to $p_{0}$, while in case of an out-of-control change the proportion is denoted by $p_{1}$.

Based on independent random samples of magnitude $n$, say $X_{1 t}, X_{2 t}, \ldots, X_{n t}, t=1,2, \ldots$, which are drawn from the process at time $t$, the variables $Y_{i t}$ and $I_{i t}$ were defined earlier. If $M_{t}$ corresponds to the total number of positive deviations $Y_{i t}$ 's, namely $M_{t}=\sum_{i=1}^{n} I_{i t}$, Riaz (2015) defined the following EWMA statistic:

$W_{t}=\lambda Q_{t}+(1-\lambda) W_{t-1}$

where $\lambda$ corresponds to the smoothing constant $(0<\lambda<1)$, while the statistic $Q_{t}$ is viewed as the average value of $M_{t-1}, M_{t}$. Given that the initial value of the above-mentioned EWMA scheme is given by $W_{0}=n p_{0}$, the corresponding mean and variance can be expressed by the aid of the following:

$$
E\left(W_{t}\right)=n p_{0}, V\left(W_{t}\right)=\lambda Q_{t}+(1-\lambda) W_{t-1},
$$

The EWMA-type nonparametric monitoring scheme can now be constructed by the aid of the following control limits:

$$
L C L=n p_{0}-\sqrt{n p_{0}\left(1-p_{0}\right)\left(\lambda /(2-\lambda)\left(1-(1-\lambda)^{2 t} / 2\right.\right.}
$$

and 
International Journal of Mathematical, Engineering and Management Sciences

Vol. 6, No. 3, 708-751, 2021

https://doi.org/10.33889/IJMEMS.2021.6.3.044

$U C L=n p_{0}+\sqrt{n p_{0}\left(1-p_{0}\right)\left(\lambda /(2-\lambda)\left(1-(1-\lambda)^{2 t} / 2\right.\right.}$

where $L$ corresponds to the multiplier utilized for mending the wrong alarms. The resulting twosided control scheme generates an alarm if the monitoring statistic $W_{t}$ defined in Eq. (36) is larger than $U C L$ or smaller than $L C L$.

Riaz (2015) appraised the attribution of the resulting control scheme by the aid of its run length. More precisely, the $A R L$, the $S D R L$, the $R A R L$, the $E Q L$ and the $P C I$ were considered in order to examine the in- and out-of-control behavior of the proposed nonparametric EWMA scheme.

According to the comparative results provided by Riaz (2015), the proposed scheme seems to outperform other competitive CUSUM and EWMA charts, while its run length curve indicated the higher probability of detection compared to other schemes. Riaz (2015) argued that the efficiency of the resulting scheme could be even more strengthened by swelling the sequence of moving sign statistics or applying runs rules.

\subsection{An Enhanced Distribution-Free EWMA Sign Scheme Utilizing Sequential Mechanism}

Riaz et al. (2019) established a distribution-free arcsine EWMA sign control scheme by implementing the so-called sequential sampling scheme (see, e.g. Wald, 1943). The sequential sampling scheme is a sampling plan in which an undefined amount of groups are examined successively, accumulating the results, until a conclusion is drawn. Note that in the framework of sequential sampling procedure, the sample magnitude is not pre-specified.

The construction of the control chart established by Riaz et al. (2019) calls for the determination of two pairs of control limits $L C L_{1}, U C L_{1}, L C L_{2}, U C L_{2}$ according to the following steps.

Step 1. A random group of magnitude $n$ is collected and the following EWMA-type monitoring statistic is calculated:

$$
E W M A_{T_{i}}=\lambda T_{i}+(1-\lambda) E W M A_{T_{i-1}}
$$

where $T$ denotes the arcsine transformation, namely $T=\sin ^{-1}(\sqrt{p})$ while $p$ corresponds to the proportion of positive deviations.

Step 2. The control limits of the resulting scheme are now calculated by the aid of the following equations

$$
L C L_{1}=\sin ^{-1}(\sqrt{0.5})-L_{1} \sqrt{\frac{n}{4} \frac{\lambda}{2-\lambda}}, U C L_{1}=\sin ^{-1}(\sqrt{0.5})+L_{1} \sqrt{\frac{n}{4} \frac{\lambda}{2-\lambda}}
$$

and

$$
L C L_{2}=\sin ^{-1}(\sqrt{0.5})-L_{2} \sqrt{\frac{n}{4} \frac{\lambda}{2-\lambda}}, U C L_{2}=\sin ^{-1}(\sqrt{0.5})+L_{2} \sqrt{\frac{n}{4} \frac{\lambda}{2-\lambda}}
$$


where $L_{1}, L_{2}$ satisfy the condition $L_{1} \geq L_{2}$. It is straightforward that the resulting scheme generates an out-of-control alarm if one of the following occurs:

$$
E W M A_{T_{i}} \geq U C L_{1} \text { or } E W M A_{T_{i}} \leq L C L_{1}
$$

on the other hand, if:

$$
L C L_{2} \leq E W M A_{T_{i}} \leq U C L_{2}
$$

then the process is characterized as in-control. Of course, the case where the monitoring statistic is located between the two upper control limits $U C L_{1}, U C L_{2}$ or between the two lower control limits $L C L_{1}, L C L_{2}$, is not covered by the above-mentioned conditions stated in (42) and (43). In such case, the proposed procedure calls for resampling, namely a separate random sample of the same magnitude is collected from the process and the EWMA-type statistic is calculated once again by the aid of formula given above. Riaz et al. (2019) carried out a numerical comparative analysis among control charts which utilize single, double, repetitive and sequential sampling schemes. For illustration purposes, a real-data problem referring to smartphone accelerometer data is presented in some detail (see also Riaz et al., 2017).

The attribution of the proposed monitoring scheme is appraised and contrasted to its counterparts, by the aid of the corresponding run length properties such as the $A R L$, the $M R L$ or the SDRL. According to the numerical results provided by Riaz et al. (2019), the proposed distribution-free EWMA-type monitoring scheme is proved to be efficient in tracking down slight changes of the underlying process distribution.

\subsection{A Distribution-Free Adaptive EWMA Sign Control Chart}

Tang et al. (2019b) introduced a nonparametric adaptive EWMA control scheme for count data. Generally speaking, the adaptive EWMA schemes combine the well-known Shewhart-type and the traditional EWMA framework in a smooth way (see, e.g. Capizzi and Masarotto, 2003; Tang et al., 2019a; Aly et al., 2017). Their proposed chart is suitable for monitoring count data and consequently the corresponding plotting statistic takes on only integer values, while the respective weights should be positive integer-valued. A performance analysis based on exact run length properties is carried out by implementing an appropriate discrete-time Markov chain technique.

The nonparametric control scheme proposed by Tang et al. (2019b) utilizes the following adaptive EWMA scheme for count data.

$$
\left(\gamma_{t}+\gamma_{y}\right) Y_{t}+R_{t}=\varphi\left(e_{t}\right)+\left(\gamma_{x}+\gamma_{y}\right) Y_{t-1}+R_{t-1}
$$

where $\gamma_{t}, \gamma_{y}$ are two fixed positive integer-valued parameters, $\varphi\left(e_{t}\right)=\varphi\left(S N_{t}-Y_{t-1}\right)$ is a score function defined as ( $k$ is a positive integer-valued parameter). 
$\varphi(e)= \begin{cases}e\left(\gamma_{x}+\gamma_{y}\right)+k \gamma_{y}, & \text { if } e<-k \\ e \gamma_{x}, & \text { if }|e| \leq k \\ e\left(\gamma_{x}+\gamma_{y}\right)-k \gamma_{y}, & \text { if } e>k .\end{cases}$

Moreover, as already mentioned earlier $S N_{t}=\sum_{j=1}^{n} \operatorname{sign}\left(X_{(t) j}-\mu_{0}\right)$ (see, also Eq. (29) of the present manuscript) and

$$
C_{t}=\left(\gamma_{t}+\gamma_{y}\right) Y_{t}+R_{t}
$$

Note that the term $Y_{t}$ can be viewed as the rounded towards zero integer part of the quotient of the Euclidean division $Y_{t}=\left(\varphi\left(S N_{t}-Y_{t-1}\right)+C_{t}\right) /\left(\gamma_{x}+\gamma_{y}\right)$, where the quantity $R_{t}=\varphi\left(S N_{t}-Y_{t-1}\right)+C_{t-1}-\left(\gamma_{x}+\gamma_{y}\right) Y_{t}$, simply takes on values from the set $\left\{-\gamma_{x}-\gamma_{y}+1, \ldots, \gamma_{x}+\gamma_{y}-1\right\}$. It is straightforward that the proposed nonparametric EWMA control scheme proposed by Tang et al. (2019b) produces an out-of-control signal if $Y_{t} \geq h$ or $Y_{t} \leq-h$, where the positive design parameter $h$ is appropriately determined for accomplishing a required degree of attribution. Note that under the assumption $k \rightarrow \infty$, the proposed scheme coincides with the distribution-free scheme introduced by Castagliola et al. (2019) (see also the subsection 2.2 of the present manuscript).

\subsection{A Nonparametric Synthetic EWMA Control Chart Based on Signs}

Haq (2019) proposed a synthetic EWMA sign scheme by integrating the existing and well-known EWMA sign scheme with the conventional run length scheme, which was first introduced by Bourke (1991) based on data on individual items for controlling a specific characteristic. The socalled conforming run length chart has been already mixed with Shewhart-type, CUSUM-type and EWMA-type monitoring schemes resulting in several synthetic control charts (see, e.g. Wu and Spedding, 2000; Scariano and Calzada, 2009).

Generally speaking, in an one hundred percent investigation process, the conforming run length $(C R L$, hereafter) is viewed as the global amount of inspected observations between the two successive nonconforming observations - including the ending nonconforming unit. Denoting by $p$ the proportion of the nonconforming units, it is readily deduced that the corresponding expected value equals to $E(C R L)=1 / p$, for $C R L=1,2, \ldots$.

Haq (2019) utilized the test statistic $M=\sum_{j=1}^{n} I_{j}$, mentioned earlier (see also Eq. (16) of the present manuscript) and applied the well-known arcsine transformation under the assumption that the magnitude of random samples drawn from the process is equal to or larger than 8. In simple words, Haq (2019) recorded the statistic $Q_{t}=\sin ^{-1}(\sqrt{p})$ at time $t$ and afterwards the following EWMAtype scheme is readily obtained 
$H_{t}=\lambda Q_{t}+(1-\lambda) H_{t-1}$

where the starting value is set to be equal to $H_{0}=\sin ^{-1}(\sqrt{1 / 2})$. The control limits of the resulting nonparametric control chart are given below:

$$
L C L=\sin ^{-1}(\sqrt{1 / 2})-\frac{k}{2 \sqrt{n}} \sqrt{\frac{\lambda}{2-\lambda}}, U C L=\sin ^{-1}(\sqrt{1 / 2})+\frac{k}{2 \sqrt{n}} \sqrt{\frac{\lambda}{2-\lambda}}
$$

The step-by-step testing procedure of the resulting monitoring scheme could now be boiled down as follows:

(i) If at time $t$ the observed value of the EWMA-type statistic $H_{t}$ based on the corresponding random sample of magnitude $n$ lies between the control limits defined in Eq. (48), then the particular sample is declared to be a conforming sample and another random sample of same magnitude should be drawn. However, in different case the monitoring procedure goes on to the next stage.

(ii) The number of groups between the ongoing and the last nonconforming group is counted, namely the observed $C R L$ sample of the corresponding control chaschemert is calculated.

(iii) Once the value $C R L$ is equal to or less than $L$, then the proposed EWMA synthetic scheme generates an out-of-control alarm.

According to the run length performance comparisons provided by Haq (2019), it turns out that the resulting EWMA synthetic monitoring scheme is more sensitive than the EWMA sign scheme established by Yang et al. (2011).

\subsection{A Nonparametric EWMA Sign Control Scheme Under the Ranked Set Sampling}

Ali et al. (2020) introduced a distribution-free EWMA control scheme based on sign statistics under a ranked set sampling scheme (see, e.g. McIntyre, 1952). Some general details about the specific sampling scheme are given in subsection 2.2 of the present manuscript.

Let $Y_{q, j(h)}, q=1,2, \ldots$, denote the measurement of our concern with target value $\theta_{0}$, where each sample consists of $j=1,2, \ldots, n$ values and $h=1,2, \ldots, m$ number of cycles under the ranked set sampling scheme. In their framework, the plausible deviations of $Y$-values from $\theta_{0}$ are summarized as:

$$
\operatorname{sign}\left(Y_{q j(h)}-\theta_{0}\right)= \begin{cases}1, & \text { if } Y_{q j(h)}-\theta_{0}>0 \\ 0, & \text { if } Y_{q j(h)}-\theta_{0}=0 \\ -1, & \text { if } Y_{q j(h)}-\theta_{0}<0\end{cases}
$$

Ali et al. (2020) utilized the following EWMA-type monitoring statistic: 
International Journal of Mathematical, Engineering and Management Sciences

Vol. 6, No. 3, 708-751, 2021

https://doi.org/10.33889/IJMEMS.2021.6.3.044

$$
R E W M A_{q}=\lambda\left(R S N_{q}\right)-(1-\lambda) R E W M A_{q-1}
$$

where $\lambda$ corresponds to the smoothing constant used for assigning the weights to the ongoing and earlier observed values. The statistic $R S N_{q}$ appeared in the above-mentioned EWMA scheme, is expressed as $R S N_{q}=r^{+}-r^{-}$, where $r^{+}\left(r^{-}\right)$denotes the positive (negative) signs in a ranked set subgroup. The authors claimed that the corresponding expectation and variance are given by:

$$
E\left(R S N_{q}\right)=m n(2 p-1), \operatorname{Var}\left(R S N_{q}\right)=4 m n p(1-p) \varphi_{0}^{2}
$$

where the quantity $\varphi_{0}^{2}$ reveals the advantages of ranked set sampling over standard random sampling and is provided by Hettmansperger (1995). The control limits and the center line of the nonparametric EWMA sign monitoring scheme of Ali et al. (2020) are given below:

$$
\begin{aligned}
& L C L=m n(2 p-1)-L \sqrt{\frac{\lambda}{2-\lambda} 4 m n p(1-p) \varphi_{0}^{2}}, C L=m n(2 p-1), \\
& U C L=m n(2 p-1)+L \sqrt{\frac{\lambda}{2-\lambda} 4 m n p(1-p) \varphi_{0}^{2}}
\end{aligned}
$$

respectively. It goes without saying that the resulting nonparametric EWMA scheme generates an out-of-control alarm if $R E W M A_{q} \geq U C L$ or $R E W M A_{q} \leq L C L$. Note that the design parameters are determined appropriately for reaching a required degree of attribution.

According to some numerical results provided by the authors the in-control robustness and superiority of the resulting chart over its competitive ones in tracking down all types of changes in the process location is revealed.

\subsection{A Nonparametric Double EWMA Sign Control Chart}

Raza et al. (2020) established a distribution-free double EWMA control chart based on signed-rank statistics. More specifically, the resulting monitoring chart is constructed by melting the Wilcoxon signed-rank (SR) statistic into the EWMA framework and then combining two EWMA statistics with the motivation of enhancing the sensitivity of the resulting chart.

Let $X_{i t}, t=1,2, \ldots$, denote the $i$-th observed value of the $t$-th random sample for the quality characteristic of interest with target value $\theta_{0}$. In their framework, the singed rank statistic is defined as:

$$
S R_{t}=\sum_{i=1}^{n} I_{i t} R_{i t}^{+}, t=1,2, \ldots,
$$

where, 


$$
I_{i t}=\left\{\begin{array}{l}
1, \text { if } X_{i t}-\theta_{0}>0 \\
0, \text { if } X_{i t}-\theta_{0}=0 \\
-1, \text { if } X_{i t}-\theta_{0}<0
\end{array}\right.
$$

The plotted statistic $D E_{S R_{t}}$ of the resulting double EWMA monitoring scheme is a mixture of two EWMA statistics which utilizes the signed rank statistic defined in Eq. (52). More specifically, the proposed EWMA statistic is expressed by the aid of the following equation:

$$
D E_{S R_{t}}=\lambda_{1} E_{S R_{t}}+\left(1-\lambda_{1}\right) D E_{S R_{t-1}}, 0<\lambda_{1} \leq 1
$$

where

$$
E_{S R_{t}}=\lambda_{2} S R_{t}+\left(1-\lambda_{2}\right) E_{S R_{t-1}}, 0<\lambda_{2} \leq 1
$$

while $\lambda_{1}, \lambda_{2}$ are two smoothing constants. Note that both initial values of $D E_{S R_{t}}, E_{S R_{t}}$ are equal to zero. The control limits of the double EWMA nonparametric scheme proposed by Raza et al. (2020) are given by:

$$
\begin{aligned}
L C L=-k & \sqrt{\left(\frac{\lambda_{1} \lambda_{2}}{\lambda_{1}-\lambda_{2}}\right)^{2}\left[\sum_{i=1}^{2} \frac{\left(1-\lambda_{i}\right)^{2}\left(1-\left(1-\lambda_{i}\right)^{2 t}\right)}{1-\left(1-\lambda_{i}\right)^{2}}-\frac{2\left(1-\lambda_{1}\right)\left(1-\lambda_{2}\right)\left\{1-\left(1-\lambda_{1}\right)^{t}\left(1-\lambda_{2}\right)^{t}\right\}}{1-\left(1-\lambda_{1}\right)\left(1-\lambda_{2}\right)}\right]} \\
& \times \sqrt{\left\{\frac{n(n+1)(2 n+1)}{6}\right\}}
\end{aligned}
$$

and

$$
\begin{aligned}
U C L= & k \sqrt{\left(\frac{\lambda_{1} \lambda_{2}}{\lambda_{1}-\lambda_{2}}\right)^{2}\left[\sum_{i=1}^{2} \frac{\left(1-\lambda_{i}\right)^{2}\left(1-\left(1-\lambda_{i}\right)^{2 t}\right)}{1-\left(1-\lambda_{i}\right)^{2}}-\frac{2\left(1-\lambda_{1}\right)\left(1-\lambda_{2}\right)\left\{1-\left(1-\lambda_{1}\right)^{t}\left(1-\lambda_{2}\right)^{t}\right\}}{1-\left(1-\lambda_{1}\right)\left(1-\lambda_{2}\right)}\right]} \\
& \times \sqrt{\left\{\frac{n(n+1)(2 n+1)}{6}\right\}}
\end{aligned}
$$

respectively. Note that parameter $k$ simply expresses how many times the standard deviation of the monitoring statistic wriggles into the decision interval of the resulting chart.

It is forthright that the proposed distribution-free double EWMA chart produces an out-of-control alarm if $D E_{S R_{t}}>U C L$ or $D E_{S R_{t}}<L C L$. Raza et al. (2020) also provided an algorithm for computing several run length characteristics of the proposed EWMA scheme under several distributional assumptions, such as Laplace, Logistic, Standard Normal, Student's $t$ or contaminated Normal distributional model. Raza et al. (2020) considered a real industrial dataset of a combined cycle power plant in order to offer some practical implementation of the resulting double EWMA nonparametric control chart. 
International Journal of Mathematical, Engineering and Management Sciences

Vol. 6, No. 3, 708-751, 2021

https://doi.org/10.33889/IJMEMS.2021.6.3.044

\subsection{A Distribution-Free Repetitive Sampling DEWMA Scheme Based on Linear Prediction}

Shafqat et al. (2020) introduced a linear prediction related double EWMA sign control scheme utilizing the repetitive sampling plan. The resulting control scheme is suitable for monitoring binomially distributed data and seems to be efficient in tracking down plausible small changes of the underlying process.

Following a similar argumentation as the one provided by Brown and Meyer (1961), Shafqat et al. (2020) constructed a double EWMA control chart for delivering linear predictions of the form $F_{(i+t)}=a_{i}+b_{i} t$, where $F_{(i+t)}$ corresponds to the forecast in the period $t$ while the coefficients $a_{i}$ and $b_{i}$ are given as:

$a_{i}=2 Z_{i}-Z_{i}^{\prime}, b_{i}=\frac{\lambda}{1-\lambda}\left(Z_{i}-Z_{i}^{\prime}\right)$

Note that $Z_{i}$ is the arcsine-transformed EWMA sign statistic mentioned earlier, while $Z_{i}^{\prime}$ corresponds to the monitoring statistic of the respective double EWMA control chart (see, e.g. Shamma and Shamma, 1992).

The asymptotic control limits of nonparametric linear prediction-based double EWMA control chart designed for repetitive sampling are determined by the aid of the following equations:

$$
\begin{gathered}
U C L_{1}=\sin ^{-1} \sqrt{p_{0}}+k_{1} \sqrt{\operatorname{Var}\left(F_{t}\right)}, L C L_{1}=\sin ^{-1} \sqrt{p_{0}}-k_{1} \sqrt{\operatorname{Var}\left(F_{t}\right)}, \\
C L=\sin ^{-1} \sqrt{p_{0}}, \\
U C L_{2}=\sin ^{-1} \sqrt{p_{0}}+k_{2} \sqrt{\operatorname{Var}\left(F_{t}\right)}, L C L_{2}=\sin ^{-1} \sqrt{p_{0}}-k_{2} \sqrt{\operatorname{Var}\left(F_{t}\right)},
\end{gathered}
$$

where

$$
\operatorname{Var}\left(F_{t}\right)=\frac{1}{4 n} \frac{\lambda\left(1+4(1-\lambda)+5(1-\lambda)^{2}\right)}{(1+(1-\lambda))^{2}}+\frac{2 \lambda^{3}(1+3(1-\lambda))}{(1+(1-\lambda))^{3}}+\frac{\lambda^{2}(1+3(1-\lambda))}{(1+(1-\lambda))^{3}}
$$

the control coefficients $k_{1}$ and $k_{2}$ take on values depending on the other design parameters of the scheme as well as its required in- or out-of-control level of attribution. The nonparametric chart proposed by Shafqat et al. (2020) declares that the process is in-control if $L C L_{2} \leq F_{t} \leq U C L_{2}$, while whenever $U C L_{2} \leq F_{t}<U C L_{1}$ or $L C L_{1}<F_{t} \leq L C L_{2}$, the process is repeated. A real-life application based on volume measurements of soft drink bottles (see, e.g. Montgomery, 2009).

\subsection{A Nonparametric EWMA Control Scheme for Monitoring Time-Between- Events-And-Amplitude Data}

$\mathrm{Wu}$ et al. (2020) established nonparametric control chart for joint monitoring the time interval $T$ between successive occurrences of an event $E$ and its magnitude $X$. The authors also established a "continuousify" technique combined with a common Markov chain technique in order to 
International Journal of Mathematical, Engineering and Management Sciences

Vol. 6, No. 3, 708-751, 2021

https://doi.org/10.33889/IJMEMS.2021.6.3.044

investigate the run length distribution and its characteristics. It is evident that several schemes which are suitable for monitoring both the time interval $T$ and the magnitude $X$ have already been studied in the literature (see, e.g. Wu et al., 2009; Qu et al., 2018; Rahali et al., 2019).

Let $D_{0}=0, D_{1}, D_{2}, \ldots$ be the dates of occurrence of a specific negative event $E$, while $T_{1}=D_{1}-D_{0}, T_{2}=D_{2}-D_{1}, \ldots$ denote the time intervals between two successive occurrences of the event $E$. If $X_{1}, X_{2}, \ldots$ are the respective magnitudes of this event occurring at times $D_{1}, D_{2}, \ldots$, then it is presumed that $X_{1}, X_{2}, \ldots$ are independent of $T_{1}, T_{2}, \ldots$. Denoting by $\theta_{T}, \theta_{X}$ two known $a$ quantiles of the corresponding distributions of the continuous variables $T$ and $X$, Wu et al. (2020) defined the following sign statistics:

$$
S T_{i}=\operatorname{sign}\left(T_{i}-\theta_{T_{0}}\right), S X_{i}=\operatorname{sign}\left(X_{i}-\theta_{X_{0}}\right)
$$

where $\theta_{T_{0}}, \theta_{X_{0}}$ are the respective in-control values. Based on the above-mentioned sign statistics, the statistic $S_{i}$ can now be readily defined as:

$$
S_{i}=\left(S X_{i}-S T_{i}\right) / 2
$$

where $S_{i}$ takes on values $-1,0$ and 1 when the process is in an acceptable, unacceptable and intermediate situation respectively. The issue of this method that should be appropriately addressed is that, because of the discrete nature of the random variable $S_{i}$, it is impossible to accurately compute the run length properties under such framework. Consequently, Wu et al. (2020) proposed to transform the discrete random variable $S_{i}$ into a new continuous one, denoted as $S_{i}^{*}$. More precisely, the random variable $S_{i}^{*}$ is defined as a mixture of three normally distributed variables and a proper Markov chain technique is now implemented for investigating the run length distribution of the proposed nonparametric monitoring scheme.

Wu et al. (2020) proposed an upper-sided EWMA-type control scheme for tracking down plausible increase in $S_{i}, S_{i}^{*}$. Their proposed nonparametric monitoring scheme is based on the following statistic:

$$
Z_{i}^{*}=\max \left(0, \lambda S_{i}^{*}+(1-\lambda) Z_{i-1}^{*}\right), 0<\lambda<1
$$

while the corresponding upper control limit is given by:

$$
U C L=k \sqrt{\frac{\lambda\left(\sigma^{2}+0.5\right)}{2-\lambda}}, k>0
$$

For illustration purposes, Wu et al. (2020) studied a real data set concerning the time (in days) between fires in forests of a French region and their magnitudes. 
International Journal of Mathematical, Engineering and Management Sciences

Vol. 6, No. 3, 708-751, 2021

https://doi.org/10.33889/IJMEMS.2021.6.3.044

\subsection{A Nonparametric Sign Control Chart Based on A Controlled EWMA Statistic}

Aslam et al. (2020) established a nonparametric sign control scheme which relies on the modified or controlled EWMA statistic. More precisely, the indicator $I_{j}$ defined in Eq. (16) of the present manuscript is utilized, while the random sum $M=\sum_{j=1}^{n} I_{j}$, which follows the binomial distribution with parameters $n$ and $p=0.5$, under the presumption that the process is in-control is also considered. Aslam et al. (2020) proposed a modified EWMA monitoring scheme by involving further controlled term in the EWMA sign statistic utilized by Yang et al. (2011). The proposed controlled modified EWMA-type sign statistic is expressed as:

$$
M E W M A_{M_{i}}=\lambda M_{i}+(1-\lambda) M E W M A_{M_{i}}+k\left(M_{i}-M_{i-1}\right),-1<k<0
$$

Note that the proposed modified EWMA scheme defined in Eq. (60) reduces to the EWMA sign monitoring scheme of Yang et al. (2011) if we set $k$ equal to zero.

The control limits of the two-sided control chart proposed by Aslam et al. (2020) are given below:

$$
L C L=n / 2-L \sqrt{\frac{\left(\lambda+2 \lambda k+2 k^{2}\right) n}{4(2-\lambda)}}, U C L=n / 2+L \sqrt{\frac{\left(\lambda+2 \lambda k+2 k^{2}\right) n}{4(2-\lambda)}}
$$

It is forthright that the proposed distribution-free modified EWMA sign chart generates an out-ofcontrol alarm if $M E W M A_{M_{i}}>U C L$ or $M E W M A_{M_{i}}<L C L$.

The resulting scheme is contrasted to other distribution-free EWMA sign control schemes. According to the numerical results provided by the same authors, one may readily deduce that the resulting scheme is more sensitive than the competitive EWMA sign scheme in terms of run length characteristics. Moreover, Aslam et al. (2020) argued that the resulting control scheme is an attractive alternative for practitioners, especially when the aim is to track down slight to middling changes in the process.

\subsection{A Nonparametric Double Generally Weighted Moving Average Signed-Rank Control Chart}

Alevizakos et al. (2020b) established a nonparametric double GWMA control scheme based on the signed-rank statistic for monitoring the location parameter of the underlying process. The run length distribution of the resulting chart is investigated in some detail, while plenty numerical calculations have been accomplished based on proper Monte Carlo simulation procedures.

Let $X_{i t}, t=1,2, \ldots$, denote the $i$-th observed value of the $t$-th random sample for the quality characteristic of interest with target value $\theta_{0}$. In their framework, the signed rank statistic $S R$ defined earlier is recalled (see, e.g. Eq. (52) of the present manuscript) in order to define the charting statistic of the proposed double GWMA scheme via the system of the following equations: 


$$
\left\{\begin{array}{l}
G_{t}=\sum_{j=1}^{t}\left(q_{1}^{(j-1)^{a}}-q_{1}^{j^{a}}\right) S R_{t-j+1} \\
D G_{t}=\sum_{j=1}^{t}\left(q_{2}^{(j-1)^{\beta}}-q_{1}^{j^{\beta}}\right) G_{t-j+1}
\end{array}\right.
$$

where $0 \leq q_{1}, q_{2}<1$ are design constants of the resulting scheme and $a, \beta$ correspond to the adjustment parameters determined appropriately by the practitioner. The time-varying control limits of the two-sided monitoring scheme proposed by Alevizakos et al. (2020b) are given below:

$$
L C L_{t}=-L \sqrt{\frac{n(n+1)(2 n+1)}{6} W_{t}}, U C L_{t}=L \sqrt{\frac{n(n+1)(2 n+1)}{6} W_{t}}
$$

where $W_{t}=\sum_{j=1}^{t} w_{j}^{2}$, is the sum of squares of the weights and $L$ corresponds to the width of control limits. It is evident that for large values of $t$, the asymptotic or steady-state control limits of the proposed monitoring scheme can be written as:

$L C L=-L \sqrt{\frac{n(n+1)(2 n+1)}{6} W}, U C L=L \sqrt{\frac{n(n+1)(2 n+1)}{6} W}$

where, $W=\lim _{t \rightarrow \infty} W_{t}$.

It is forthright that the resulting nonparametric sign scheme generates an alarm if the charting statistic is located out of the region determined by the corresponding control limits.

According to the extensive numerical results provided by Alevizakos et al. (2020b), the proposed double GWMA monitoring scheme seems to be more efficient than its competitors for slight changes in the location parameter, while its attribution is similar to the other nonparametric charts for bigger changes.

\subsection{A Distribution-Free Triple EWMA Sign Control Scheme}

Alevizakos et al. (2020a) proposed a triple EWMA control scheme which utilizes the sign statistic for monitoring the location parameter of the underlying process. The run length-based attribution characteristics of the resulting scheme are studied in detail, while several numerical comparisons revealed its superiority against other competitors, especially for slight changes. The indicator defined in Eq. (16) of the present manuscript is utilized, while the random binomial variable $N_{t}=\sum_{i=1}^{n} I_{i t}$, with parameters $n$ and $p=0.5$, under the presumption that the process is in-control is also considered. The charting statistic of the proposed triple EWMA scheme can be evaluated through the system of the following equation: 


$$
\left\{\begin{array}{l}
Z_{t}=\lambda N_{t}+(1-\lambda) Z_{t-1} \\
D_{t}=\lambda Z_{t}+(1-\lambda) D_{t-1} \\
W_{t}=\lambda D_{t}+(1-\lambda) W_{t-1}
\end{array}\right.
$$

where $0<\lambda<1$ is the smoothing parameter of the resulting chart while the initial values are set to be equal to $n / 2$. The time-varying control limits of the two-sided monitoring scheme proposed by Alevizakos et al. (2020a) are given below:

$$
L C L_{t}=n / 2-L \sqrt{\operatorname{Var}_{i n}\left(W_{t}\right)}, U C L_{t}=n / 2+L \sqrt{\operatorname{Var}_{i n}\left(W_{t}\right)}
$$

where the corresponding center line is given as $C L=n / 2$, while the in-control variance of the statistic $W_{t}$ appeared in Eq. (66) can be computed by the aid of the following formula:

$$
\begin{gathered}
\operatorname{Var}_{\text {in }}\left(W_{t}\right)=\left[\frac { d ^ { 3 } \lambda ^ { 6 } } { 4 } \left[-\frac{t\left(t^{2}-1\right)(t-2) d^{t-3}}{1-d}-\frac{4 t\left(t^{2}-1\right) d^{t-2}}{(1-d)^{2}}-\frac{12 t(t+1) d^{t-1}}{(1-d)^{3}}\right.\right. \\
\left.-\frac{24(t+1) d^{t}}{(1-d)^{4}}+\frac{24\left(1-d^{t+1}\right)}{(1-d)^{5}}\right]+2 d^{2} \lambda^{6}\left[\frac{t\left(t^{2}-1\right)(t-2) d^{t-2}}{1-d}\right. \\
\left.-\frac{3 t(t+1) d^{t-1}}{(1-d)^{2}}-\frac{6(t+1) d^{t}}{(1-d)^{3}}+\frac{6\left(1-d^{t+1}\right)}{(1-d)^{4}}\right] \\
+\frac{7 d \lambda^{6}}{2}\left[-\frac{t(t+1) d^{t-1}}{1-d}-\frac{2(t+1) d^{t}}{(1-d)^{2}}+\frac{2\left(1-d^{t+1}\right)}{(1-d)^{3}}\right] \\
\left.+\lambda^{6}\left[\frac{1-d^{t+1}}{(1-d)^{2}}-\frac{(t+1) d^{t}}{1-d}\right]\right] \frac{n}{4}
\end{gathered}
$$

It is evident that for large values of $t$, the asymptotic or steady-state control limits of the proposed monitoring scheme can be written as:

$$
\begin{aligned}
& L C L=n / 2-L \sqrt{\left(\frac{6(1-\lambda)^{6} \lambda}{(2-\lambda)^{5}}+\frac{12(1-\lambda)^{4} \lambda^{2}}{(2-\lambda)^{4}}+\frac{7(1-\lambda)^{2} \lambda^{3}}{(2-\lambda)^{3}}+\frac{\lambda^{4}}{(2-\lambda)^{2}}\right) \frac{n}{4}}, \\
& U C L=n / 2+L \sqrt{\left(\frac{6(1-\lambda)^{6} \lambda}{(2-\lambda)^{5}}+\frac{12(1-\lambda)^{4} \lambda^{2}}{(2-\lambda)^{4}}+\frac{7(1-\lambda)^{2} \lambda^{3}}{(2-\lambda)^{3}}+\frac{\lambda^{4}}{(2-\lambda)^{2}}\right) \frac{n}{4}}
\end{aligned}
$$

It is forthright that the proposed distribution-free scheme generates an out-of-control alarm if the charting statistic is located out of the region determined by the corresponding control limits. 
According to the extensive numerical results provided by Alevizakos et al. (2020b), the proposed double GWMA control scheme seems to be more efficient than its competitors for slight changes in the location parameter, while its attribution seems to be almost similar compared to the other nonparametric charts for bigger changes.

\subsection{A Nonparametric EWMA Control Chart Based on Auxiliary Information}

Haq (2020) introduced a nonparametric EWMA sign control chart by the aid of an auxiliaryinformation-based estimator of the process mean. The run length characteristics of the proposed scheme are investigated via an appropriate Markov chain approach. Let $Y$ be the measurement of our concern with target value $\mu_{Y}$, while the probability of observing a $Y$-value greater than $\mu_{Y}$ is denoted by $p$. Suppose next that there is an auxiliary characteristic $X$ being related to the measurement $Y$. It is also presumed that the underlying joint probability distribution of $(Y, X)$ is unascertained in contrast to the population parameters. Denoting by $\mu_{X}, \mu_{Y}, \sigma_{X}, \sigma_{Y}$ the population means and variances of $X$ and $Y$, an unbiased estimator $Y^{*}$ of the process mean can be determined by the aid of the following formula (see, e.g. Cochran, 2007).

$Y^{*}=Y+\rho \frac{\sigma_{X}}{\sigma_{Y}}\left(\mu_{X}-X\right)$

where $\rho$ corresponds to the correlation coefficient between $X$ and $Y$. The center line and the control limits of the two-sided monitoring scheme proposed by Haq (2020) are given below:

$$
\begin{aligned}
& U C L=\sin ^{-1} \sqrt{p_{0}}+\frac{k}{2 \sqrt{n}} \sqrt{\frac{\lambda}{2-\lambda}}, L C L=\sin ^{-1} \sqrt{p_{0}}-\frac{k}{2 \sqrt{n}} \sqrt{\frac{\lambda}{2-\lambda}}, \\
& C L=\sin ^{-1} \sqrt{p_{0}},
\end{aligned}
$$

where $k$ is a positive design parameter which is determined appropriately in order to achieve a required degree of in- and out-of-control behavior. Haq (2020) illustrated the proposed chart for monitoring simulated bivariate data based on the Plackett's distribution.

\section{Nonparametric EWMA Monitoring Schemes Based On Rank Statistics}

In the present section, we refer to univariate nonparametric schemes, which rely on ranks and follow the general EWMA framework. It is evident that rank-based statistics have been already utilized for constructing Shewhart-type distribution-free control schemes (see, e.g. Triantafyllou and Panayiotou, 2020; Triantafyllou, 2021). However, our study aims at discussing the corresponding EWMA -type advances.

We next provide a detailed review of nonparametric univariate monitoring schemes based on ranks, namely of these members of the EWMANCC class which employ several rank-based statistics. The set-up of presentation of the included articles is determined to be chronological for the purpose of setting up a comprehensible and well-organized flux. 
International Journal of Mathematical, Engineering and Management Sciences

Vol. 6, No. 3, 708-751, 2021

https://doi.org/10.33889/IJMEMS.2021.6.3.044

\subsection{A Nonparametric EWMA Control Chart Based on Standardized Ranks}

Hackl and Ledolter (1991) introduced a nonparametric framework by considering the exponentially weighted moving means of the ranks of the observations. The resulting chart seems to be outlierresistant and performs well even if a sudden change of the process mean is occurred. Let $X_{t}, t=1,2, \ldots$, be a sequence of independent random variables from a continuous distribution with cumulative distribution function $F(x)$. The so-called standardized rank $R_{t}$ of the observation $X_{t}$ is defined as:

$R_{t}=2\left(F_{0}\left(X_{t}\right)-0.5\right)$

where $F_{0}(x)$ denotes the in-control distribution of the underlying production. As Hackl and Ledolter (1991) argued, the standardized rank $R_{t}$ is uniformly distributed, while it takes on values between -1 and 1 with mean and variance equal to zero and 1/3 respectively. In case of an unknown in-control distribution, one may take advantage of an available historical in-control reference sample of magnitude $(g-1)$, say $Y_{1}, Y_{2}, \ldots, Y_{g-1}$, and afterwards the standardized rank of $X_{t}$ is defined as:

$$
R_{t}=\frac{2}{g}\left(R_{t}^{*}-\frac{g+1}{2}\right)
$$

where $R_{t}^{*}$ denotes the rank of $X_{t}$ in the reference sample. In other words, the rank $R_{t}^{*}$ can be viewed as $R_{t}^{*}=1+\sum_{i=1}^{g-1} I\left(X_{t}>X_{i}\right)$, where the indicator variable $I\left(X_{t}>X_{i}\right)=1$, if $X_{t}>X_{i}$ and $I\left(X_{t}>X_{i}\right)=0$, if $X_{t}<X_{i}$. The EWMA-type monitoring statistic proposed by Hackl and Ledolter (1991) is given by:

$T_{t}=(1-\lambda) T_{t-1}+\lambda R_{t}, t=1,2, \ldots$

where the smoothing parameter $\lambda$ takes on values between 0 and 1 . It is readily observed that the monitoring statistic corresponds to a weighted average of previous ranks, where the weights given to the ranks diminish with their age. It is forthright that the proposed distribution-free one-sided EWMA chart generates an out-of-control alarm if the charting statistic defined in Eq. (73) is greater than a suitably chosen control limit $h$.

\subsection{A Nonparametric EWMA Control Chart Based on Sequential Ranks}

Hackl and Ledolter (1992) proposed a nonparametric EWMA framework, which utilizes the socalled sequential ranks of the observations. Generally speaking, the sequential rank $R_{t}^{*}$ of an observed value $X_{t}$ is viewed as its rank among the most recent $g$ units, namely among $X_{t}, X_{t-1}, \ldots, X_{t-g+1}$. In other words, the sequential rank can be expressed via: 
International Journal of Mathematical, Engineering and Management Sciences

Vol. 6, No. 3, 708-751, 2021

https://doi.org/10.33889/IJMEMS.2021.6.3.044

$R_{t}^{*}=1+\sum_{i=t-g+1}^{t} I\left(X_{t}>X_{i}\right)$

where the indicator variable $I\left(X_{t}>X_{i}\right)=1$, if $X_{t}>X_{i}$ and $I\left(X_{t}>X_{i}\right)=0$, if $X_{t}<X_{i}$. The EWMA-type monitoring statistic proposed by Hackl and Ledolter (1992) is given by:

$T_{t}=(1-\lambda) T_{t-1}+\lambda R_{t}^{(g)}, t=1,2, \ldots$

where the smoothing parameter $\lambda$ takes on values between 0 and 1 , while the initial value of the above-mentioned scheme is set to be equal to zero. Note that the quantity $R_{t}^{(g)}$ appeared in Eq. (75) corresponds to the so-called standardized sequential rank of the observation $X_{t}$, which is defined as:

$R_{t}^{(g)}=\frac{2}{g}\left(R_{t}^{*}-\frac{g+1}{2}\right)$

It is of some interest to notice that the distribution of variable $R_{t}^{(g)}$ is discrete. Moreover, it is of some interest to mention that if $|\lambda|$ is strictly smaller than 1 and $t$ is large, then the distribution of the statistic $T_{t}$ defined above, will not depend on the starting value anymore. It is forthright that the proposed distribution-free one-sided EWMA scheme generates an out-of-control alarm whenever the charting statistic defined in Eq. (73) is greater than a suitably chosen control limit $h$.

\subsection{Nonparametric EWMA Control Charts Based On Wilcoxon Rank-Sum Statistic} In the present subsection we shall focus on several nonparametric EWMA-type control charts, which utilize as their monitoring statistic the well-known Wilcoxon rank-sum statistic or its modifications. It is evident that the Wilcoxon rank-sum statistic has been already utilized for constructing Shewhart-type monitoring schemes (see, e.g. Balakrishnan et al., 2009; Triantafyllou and Panayiotou, 2020; Triantafyllou, 2021). However, throughout the lines of the present subsection, we shall refer only to EWMA-type schemes of the above-mentioned class.

Li et al. (2010) introduced an analog of EWMA control chart based on the Wilcoxon rank-sum statistic for tracking down plausible changes of the mean of the underlying process. Their study relies on the run length distribution of the resulting nonparametric control chart. According to their numerical results, the proposed distribution-free EWMA scheme outperforms its competitors when the underlying distribution is non-normal, while it acts almost the same when the process is supposed to be normally distributed.

Let $X_{1}, X_{2}, \ldots, X_{m}$ be a group of magnitude $m$ drawn randomly from the process when it is incontrol. The $X$-observations form the so-called reference sample of the charting procedure. Afterwards, several test samples of magnitude $n$, e.g. $Y_{1}, Y_{2}, \ldots, Y_{n}$ are drawn from the process for characterizing the status of the ongoing production. For determining the well-known Wilcoxon 
International Journal of Mathematical, Engineering and Management Sciences

Vol. 6, No. 3, 708-751, 2021

https://doi.org/10.33889/IJMEMS.2021.6.3.044

rank-sum statistic (see, e.g. Wilcoxon, 1945), one may arrange all $(m+n)$ observations in ascending order and put them in a pooled sample. Afterwards, define $a_{i}$ such that:

$a_{i}=\left\{\begin{array}{l}1, \text { if a } X-\text { observation is placed in the } i-t h \text { entry of the pooled sample } \\ 0, \text { if a } Y \text {-observation is placed in the } i-t h \text { entry of the pooled sample }\end{array}\right.$

The Wilcoxon rank-sum statistic can now be viewed as:

$W=\sum_{i=1}^{m+n} i a_{i}$

while its mean and variance under the identical distributions' assumption, are given by:

$E(W)=\frac{n(m+n+1)}{2}, \operatorname{Var}(W)=\frac{m n(m+n+1)}{12}$

respectively. Based on the above-mentioned argumentation, Li et al. (2010) proposed the following EWMA-type statistic:

$T_{i}=\lambda W_{i}+(1-\lambda) T_{i-1}, 0<\lambda \leq 1$

where $\lambda$ is the smoothing parameter of the EWMA scheme. The control limits of the resulting EWMA-type nonparametric monitoring scheme are given below:

$L C L=\frac{n(m+n+1)}{2}-h, U C L=\frac{n(m+n+1)}{2}+h$

It goes without saying that the EWMA chart produces an out-of-control signal whenever $T_{i}$ falls outside or on the control limits.

In addition, Malela-Majika et al. (2018) developed a nonparametric EWMA-type control chart based on the same rank statistic, namely the Wilcoxon rank-sum statistic. However, in their framework the so-called repetitive sampling is utilized in order to improve the sensitivity of the resulting EWMA scheme in tracking down plausible changes of the process mean.

The authors delivered theoretical formulae as well as practical algorithms to promote the structure and applicability of the resulting scheme. Moreover, its attribution is appraised by the aid of several characteristics, such as the ARL, the SDRL, the Average Sample Size or Percentiles of the Run Length.

Generally speaking, in a repetitive sampling plan, when a sample of magnitude $n$ is drawn from the process, the number of sample points outside the control limits is supervised over a fixed criterion which corresponds to the upper (or lower) control limit of the resulting chart. The process is declared to be in-control, out-of-control or inconclusive when decision on the process cannot be 
International Journal of Mathematical, Engineering and Management Sciences

Vol. 6, No. 3, 708-751, 2021

https://doi.org/10.33889/IJMEMS.2021.6.3.044

made on the basis of the first sample (see, e.g. Sherman, 1965; Balamurali and Jun, 2006; Aslam et al., 2014).

The control chart proposed by Malela-Majika et al. (2018) utilizes the monitoring EWMA-type statistic defined in Eq. (18) and its construction calls for four different control limits. The one pair of limits, say $L C L_{1}, U C L_{1}$ correspond to the outer limits, while the others $L C L_{2}, U C L_{2}$ express the so-called inner limits of the resulting chart. The control limits of the proposed chart are given below:

$$
\begin{aligned}
& L C L_{1}=\frac{n(m+n+1)}{2}-L_{1} \sqrt{\frac{\lambda}{2-\lambda}} \sqrt{\frac{m n(m+n+1)}{12}}, \\
& U C L_{1}=\frac{n(m+n+1)}{2}+L_{1} \sqrt{\frac{\lambda}{2-\lambda}} \sqrt{\frac{m n(m+n+1)}{12}}
\end{aligned}
$$

and

$$
\begin{aligned}
& L C L_{2}=\frac{n(m+n+1)}{2}-L_{2} \sqrt{\frac{\lambda}{2-\lambda}} \sqrt{\frac{m n(m+n+1)}{12}}, \\
& U C L_{2}=\frac{n(m+n+1)}{2}+L_{2} \sqrt{\frac{\lambda}{2-\lambda}} \sqrt{\frac{m n(m+n+1)}{12}}
\end{aligned}
$$

where the non-negative parameters $L_{1}, L_{2}$ correspond to the width of the control limits. The EWMAtype nonparametric control chart established by Malela-Majika et al. (2018) generates an outcontrol alarm if the monitoring statistic $T_{i}$ falls outside the outer control limits $L C L_{1}, U C L_{1}$. On the other hand, the process is characterized as in-control if the monitoring statistic stays between the inner limits $L C L_{2}, U C L_{2}$. Additionally, in case where $L C L_{1}<T_{i} \leq L C L_{2}$ or $U C L_{2} \leq T_{i}<U C L_{1}$, the next step is to draw another sample of the same magnitude form the process and calculate the EWMA statistic once again. It is straightforward that if we set $L_{1}=L_{2}$, then the monitoring scheme proposed by Malela-Majika et al. (2018) reduces to the one introduced by Li et al. (2010), whose presentation took place earlier.

Moreover, Malela-Majika (2021) considered a distribution-free EWMA-type monitoring framework, where the random variable $W$ defined earlier is applied twice. The monitoring statistic of the proposed scheme can be viewed as:

$$
Z_{t}=\lambda T_{t}+(1-\lambda) Z_{i-1}, 0<\lambda \leq 1
$$

where,

$$
T_{t}=\lambda W_{t}+(1-\lambda) T_{i-1}, 0<\lambda \leq 1
$$

Note that $T_{t}$ simply corresponds to the charting statistic of the scheme introduced by Li et al. (2010). The EWMA-type nonparametric control chart established by Malela-Majika (2021) generates an out-control alarm if the monitoring statistic $Z_{t}$ falls outside the asymptotic control limits $L C L, U C L$, where, 


$$
\begin{aligned}
& L C L=\frac{n(m+n+1)}{2}-L \sqrt{\frac{\lambda\left(2-2 \lambda+\lambda^{2}\right)}{(2-\lambda)^{3}}} \sqrt{\frac{m n(m+n+1)}{12}}, \\
& U C L=\frac{n(m+n+1)}{2}+L \sqrt{\frac{\lambda\left(2-2 \lambda+\lambda^{2}\right)}{(2-\lambda)^{3}}} \sqrt{\frac{m n(m+n+1)}{12}}
\end{aligned}
$$

Finally, Letshedi et al. (2021) proposed a new distribution-free triple EWMA control chart based on the Wilcoxon rank-sum statistic to improve the ability for tracking down plausible changes in location parameter of the underlying distribution. In their framework, a new fast initial response feature is also activated in order to make the resulting chart more efficient. Following a similar argumentation as the one established by Alevizakos et al. (2020b), Letshedi et al. (2021) constructed their triple EWMA scheme based on the following monitoring statistic:

$T_{t}=\lambda Z_{t}+(1-\lambda) T_{t-1}, 0<\lambda \leq 1$

where the statistic $Z_{t}$ is defined in Eq. (84).

The EWMA-type nonparametric control chart established by Letshedi et al. (2021) generates an alarm if the monitoring statistic $Z_{t}$ does not fall inside the control limits $L C L, U C L$, where,

$$
\begin{aligned}
& L C L=\frac{n(m+n+1)}{2}-\frac{\lambda^{3}}{2} L \sqrt{\sum_{j=1}^{t}(t-j+1)^{2}(t-j+2)^{2}(1-\lambda)^{2(t-j)}} \sqrt{\frac{m n(m+n+1)}{12}}, \\
& U C L=\frac{n(m+n+1)}{2}+\frac{\lambda^{3}}{2} L \sqrt{\sum_{j=1}^{t}(t-j+1)^{2}(t-j+2)^{2}(1-\lambda)^{2(t-j)}} \sqrt{\frac{m n(m+n+1)}{12}} .
\end{aligned}
$$

\subsection{A Sequential Rank-Based Distribution-Free Adaptive Ewma Monitoring Scheme}

Liu et al. (2013) established sequential rank-based nonparametric adaptive EWMA monitoring scheme for tracking down the unceasing change in the location parameter. Suppose that the observations $X_{t}$ are drawn independently over time from the following change-point model (see, e.g. Hawkins and Deng, 2010).

$x_{t}=\left\{\begin{array}{l}F\left(x, \mu_{0}\right), \text { for } t=1,2, \ldots, \tau \\ F\left(x, \mu_{1}\right), \text { for } t=\tau+1, \tau+2, \ldots,\end{array}\right.$

while $\tau$ is the unascertained change point parameter, $\mu_{0}\left(\mu_{1}\right)$ corresponds to the in-control (out-ofcontrol) location parameter of the process, while $F$ denotes its continuous distribution function.

We next denote by $R_{t}$ the $t$-th sequential rank, namely the rank of observed value $x_{t}$ among the observations $x_{1}, x_{2}, \ldots, x_{t}$. which can be viewed as: 


$$
R_{t}=\sum_{j=1}^{t} I\left(x_{t} \geq x_{j}\right)
$$

The mean and variance of $R_{t}$, under the presumption that the process is in-control, are given by:

$$
E\left(R_{t}\right)=(t+1) / 2, \operatorname{Var}\left(R_{t}\right)=(t+1)(t-1) / 12
$$

respectively. Since the distribution of $R_{t}$ varies as $t$ increases, it seems that the corresponding standardized values should be considered. By defining the $t$-th standardized sequential rank as:

$$
R_{t}^{*}=\frac{R_{t}-E\left(R_{t}\right)}{\sqrt{\operatorname{Var}\left(R_{t}\right)}}, t \geq 2
$$

Liu et al. (2013) introduced their adaptive nonparametric EWMA control chart, whose monitoring statistic is given by:

$$
Z_{n}=\eta R_{n}^{*}+(1-\eta) Z_{t-1}
$$

where the initial value equal to zero. Note that weight $\eta$ is actually an adaption factor and is determined via the following:

$$
\eta=1-\max \frac{1-\lambda}{\max \left\{1,\left|\bar{R}_{n, k}^{*}\right| / \omega\right\}}
$$

where $\bar{R}_{t}^{*}=k^{-1} \sum_{i=0}^{k-1} R_{n-i}^{*}$, expresses the mean of the last $k R_{t}^{*}$-values and $k, \omega, \lambda$ are predetermined constants. For illustration purposes, the above-mentioned authors implemented their proposed nonparametric control chart for monitoring real data from an aluminum electrolytic capacitor manufacturing production.

\subsection{An Adaptive Distribution-Free EWMA Control Scheme with Variable Sampling Interval}

Liu et al. (2015) introduced a nonparametric EWMA control scheme utilizing the so-called variable sampling interval for tracking down a range of changes in the location parameter of the underlying process. In their framework, the sampling interval between each pair of samples is not fixed but rather depends on what is observed in the first sample. The general idea comes from the work provided by Reynolds et al. (1988), who considered that the time interval until the next group should be short if a sample reveals some indication of a change in the process and long if there is no suspicion of a change. Consequently, Liu et al. (2015) constructed their scheme by using s a short sampling interval if the charting statistic is close to but not actually outside the control limits and a long sampling interval if it is close to target. Liu et al. (2015) utilized the monitoring statistic defined in Eq. (92) and defined the sampling time interval $D_{i}$ as: 
$D_{i}=\left\{\begin{array}{l}d_{1}, w<\left|Z_{i-1}\right|<h \\ d_{2},\left|Z_{i-1}\right| \leq w\end{array}\right.$

where $w$ is a warning limit, which is used for determining whether the sampling interval should be shortened or not, while $h$ is the control limit, which formulates the so-called action region of the underlying process. The authors appraised the attribute of the resulting EWMA monitoring scheme by the aid of well-known measures, such as the expected value of time from the beginning to the point when the chart signals (even if the process is in-control) and the expected value of the time from process change to the time when the chart signals.

In addition, Liu et al. (2021) proposed an adaptive nonparametric EWMA control chart with dynamic sampling intervals. The particular scheme can efficiently track down various magnitudes of changes and exhibits robust attribution under alternative distributional models (based on plenty simulation numerical results carried out by the authors). Moreover, the manageable computational workload confirms that this approach is applicable for monitoring big data flows. The EWMA-type monitoring scheme introduced by Liu et al. (2021) utilizes standardized sequential ranks, the test statistic defined in Eq. (92) and the so-called dynamic sampling intervals method, which does not call for specifying sampling intervals ahead of time, and may accomplish a roughly optimal attribution compared to other schemes in which the sampling intervals are presumed be fixed values.

\subsection{Nonparametric EWMA Control Schemes Based on Lepage-Type Statistics}

Mukherjee (2017) established nonparametric EWMA control charts for simultaneously supervising the location and scale parameters of the underlying process. The resulting chart relies on the utilization of the Lepage statistic, which is actually a mixture of the well-known Wilcoxon rank sum statistics $T_{1}$ and the Ansari-Bradley test statistic $T_{2}$ (see, e.g. Lepage, 1971).

Let $X_{1}, X_{2}, \ldots, X_{m}$ be a group of magnitude $m$ drawn randomly from the process when it is incontrol. The $X$-observations form the so-called reference sample of the charting procedure. Afterwards, several test samples of magnitude $n Y_{j 1}, Y_{j 2}, \ldots, Y_{j n}, j=1,2, \ldots$ are drawn from the process for clarifying if a plausible shift in the process has been taken place. Denoting by $\mu_{1}, \mu_{2}$ the mean values of random variables $T_{1}, T_{2}$ and by $\sigma_{1}, \sigma_{2}$ the corresponding standard deviations, the so-called Lepage statistic $S_{L j}^{2}, j=1,2, \ldots$ is computed for the $j$-th test sample, by the aid of the following formula:

$S_{L j}^{2}=S_{1 j}^{2}+S_{2 j}^{2}$

where

$S_{1 j}^{2}=\frac{T_{1}-\mu_{1}}{\sigma_{1}}, S_{2 j}^{2}=\frac{T_{2}-\mu_{2}}{\sigma_{2}},, j=1,2, \ldots$ 
The EWMA-type statistic of the proposed chart is given by:

$Z_{j}^{*}=\max \left\{2, Z_{j}\right\}$

where, $Z_{0}^{*}=2$ and

$$
Z_{j}=\lambda S_{j}^{2}+(1-\lambda) Z_{j-1}, j=1,2, \ldots,, 0<\lambda \leq 1
$$

The one-sided EWMA-type nonparametric control chart proposed by Mukherjee (2017) produces an out-control signal whenever the monitoring statistic $Z_{j}^{*}$ is greater than the upper control limit $U C L$. Note that $U C L$ could be either steady-state or time-varying depending on $j$.

In addition, Song et al. (2020b) investigated and compared six nonparametric EWMA charts for joint monitoring the location and scale parameters of the underlying process. All schemes considered are based on Lepage-type statistics. For instance, the authors combined two separate EWMA statistics, one based on the location component of the Lepage statistic and the other relies on the scale component. By utilizing the test statistics defined in Eq. (95), Song et al. (2020b) proposed the following EWMA statistics:

$R_{1, j}=\lambda S_{1, j}^{2}+(1-\lambda) R_{1, j-1}$,

$R_{2, j}=\lambda S_{2, j}^{2}+(1-\lambda) R_{2, j-1}, j=1,2, \ldots$

where the initial values are set to be equal to 1 . The monitoring statistic $R_{E W M A, j}$ of the proposed scheme is a max-type combination of the above-mentioned statistics, namely

$R_{E W M A, j}=\max \left\{R_{1, j}, R_{2, j}\right\}, j=1,2, \ldots$

Moreover, Song et al. (2020a) introduced the concept of an EWMA monitoring scheme with a dynamic fast initial feature (FIR, hereafter) that restricts initial false alarms and also improves the out-of-control attribution of the resulting framework. More precisely, they established distributionfree FIR-based EWMA monitoring frameworks which rely on either the Lepage or the Cucconi statistic. The resulting charts track down changes little later than the conventional EWMA schemes when the process change occurs at a later stage and not in the early stage of monitoring. However, the proposed FIR-based nonparametric control charts seem to be beneficial for tracking down a process deviation at the zero-state or for a change at an early stage of monitoring.

\subsection{Nonparametric EWMA Control Schemes for Monitoring Time Between Events and Event Magnitude}

Huang et al. (2018) established two nonparametric EWMA control charts for joint monitoring of time between events and event magnitude. Their proposed schemes (JK chart and MA chart, 
hereafter) are based on the so-called Mathur statistic and Jurečková and Kalina statistic respectively (Mathur, 2009; Jurečková and Kalina, 2012).

Let $\mathbf{S}=\left(V_{1}, \ldots, V_{m+n}\right)$ be the pooled group of $\mathbf{S}_{m}^{(0)}, \mathbf{S}_{n}^{(1)}$, where $V_{p}=V_{p}^{(0)}, p=1,2, \ldots, m$, $V_{q}=V_{q}^{(1)}, q=1,2, \ldots, n$. In order to construct the test statistic based on the ranks of distances, the Euclidean norm $\left\{d_{i j}=V_{i} V_{j}, i=1,2, \ldots, m+n, j=1,2, \ldots, m+n, i \neq j\right\}$ is utilized as the distance metric. Consider a set of $r$ random samples from $m$ reference samples as the benchmarks. For a fixed $i$, the ranks of $d_{i k}$ in the pooled samples can be expressed as:

$$
\left(R_{i, r+1}, R_{i, r+2}, \ldots, R_{i, k}, \ldots, R_{i, m+n}\right), r+1 \leq k \leq m+n .
$$

The bivariate Wilcoxon-type rank statistic proposed by Jurečková and Kalina (2012) is given by:

$$
U_{i}=\sum_{k=m+1}^{m+n} R_{i k}, i=1,2, \ldots, r
$$

Consequently, Huang et al. (2018) utilized the following statistic:

$$
W_{j}^{*}=\frac{1}{r} \sum_{i=1}^{r} W_{i}^{2}
$$

where $W_{i}$ corresponds to the standardized value of $U_{i}$. The EWMA-type monitoring statistic of the proposed JK chart is given by:

$$
S_{j}=\lambda W_{j}^{*}+(1-\lambda) S_{j-1}, j=1,2, \ldots
$$

where $\lambda$ corresponds to the smoothing parameter and $S_{0}=1$. The one-sided JK control chart generates an alarm if the monitoring statistic $S_{j}$ exceeds the upper control limit $H$.

On the other hand, Huang et al. (2018) considered also the Mathur's statistic. Let $Y_{0 i}^{2}=\left(X_{i}^{(0)}\right)^{2}+\left(Y_{i}^{(0)}\right)^{2}, i=1,2, \ldots, m$ and $Y_{1 j}^{2}=\left(X_{j}^{(1)}\right)^{2}+\left(Y_{j}^{(1)}\right)^{2}, j=1,2, \ldots, n$. The so-called MA statistic is defined as:

$$
M A=\sum_{j=1}^{n} \sum_{i=1}^{m} L_{i j}
$$

where

$$
L_{i j}=\left\{\begin{array}{l}
1, \text { if } Y_{0 i}^{2}>Y_{1 j}^{2} \\
0, \text { otherwise }
\end{array}\right.
$$


International Journal of Mathematical, Engineering and Management Sciences

Vol. 6, No. 3, 708-751, 2021

https://doi.org/10.33889/IJMEMS.2021.6.3.044

The EWMA-type monitoring statistic of the proposed MA chart is given by:

$S_{M A, j}=\lambda M A_{j}+(1-\lambda) S_{M A, j-1}, j=1,2, \ldots$

where, $\lambda$ corresponds to the smoothing parameter and $S_{M A, 0}=m n / 2$.

The two-sided MA control chart generates an alarm if the monitoring statistic $S_{M A, j}$ falls outside the control limits $L C L, U C L$, where

$L C L=m n / 2-L \sqrt{\frac{m n}{12}(m+n+1)}, U C L=m n / 2+L \sqrt{\frac{m n}{12}(m+n+1)}$

Both EWMA schemes established by Huang et al. (2018) perform better than their corresponding Shewhart schemes especially for slight to middling changes. The resulting JK scheme performs more efficiently than the corresponding MA chart in most cases apart from the case when location changes of asymmetric distributions come from same directions. Moreover, the MA EWMA chart meets some $A R L$-biased issues when the changes come from different directions. By the aid of an extensive simulation investigation, Huang et al. (2018) deduced that the JK chart is sensitive in monitoring different combinations of changes with same or different directions and still performs well even for highly tailed distributions and highly asymmetric distributions.

\subsection{A Nonparametric EWMA Control Chart Based on Hogg-Fisher-Randle Statistic}

Mukherjee et al. (2019) introduced EWMA-type distribution-free control schemes based on the socalled Hogg-Fisher-Randles (HFR, hereafter) statistic in order to investigate slight and tenacious changes in particular measurements of the underlying production. Gastwirth (1965) increased the efficiency of rank-based testing procedures by sacrificing some ranks of the combined samples. Based on this idea, Hogg et al. (1975) developed the so-called HFR statistic, which could be viewed as an alternative to the Wilcoxon rank-sum statistic defined earlier, for addressing the two-sample location problem, especially in the case of heavy-tailed distributions. Note that the abovementioned HFR test statistic has been already utilized in the literature (see, e.g. Mukherjee and Sen, 2015).

Let $X_{1}, X_{2}, \ldots, X_{m}$ be a sample of magnitude $m$ drawn randomly from the process when it is incontrol. The $X$-observations form the so-called reference sample of the charting procedure. Afterwards, a lot of test samples of magnitude $n Y_{j 1}, Y_{j 2}, \ldots, Y_{j n}, j=1,2, \ldots$ are drawn from the process in order to decide whether the process is still in-control or a change in either the location or the scale parameter has been occurred. Denoting by $R_{i j}$ the rank of observations $Y_{i j}$ in the combined group of magnitude $(m+n)$, the $H F R$ statistic $T_{\mathrm{HFR}, i}$ can be computed through the next formula:

$T_{H F R, i}=\sum_{j: R_{i j} \leq K} R_{i j}, \quad$ for $[(m+n+1) / 2] \leq K \leq m+n$ 
International Journal of Mathematical, Engineering and Management Sciences

Vol. 6, No. 3, 708-751, 2021

https://doi.org/10.33889/IJMEMS.2021.6.3.044

where $[x]$ corresponds to the greatest integer contained in $x$. Since Hogg et al. (1975) considered the sum of ranks of only those $Y$ samples, whose ranks are less than or equal to the median rank of the combined samples, the HFR statistic seems to be suitable for tracking down plausible changes in location parameter in right-skewed distributions.

The in-control mean and variance of HFR test statistic are given by (see, e.g. Hogg et al., 1975):

$$
E\left(T_{H F R}\right)=\frac{n K(K+1)}{2(m+n)}, V\left(T_{H F R}\right)=\frac{m n K(K+1)\left(-3 K^{2}+K(4(m+n)-3)+2(m+n)\right)}{12(m+n-1)(m+n)^{2}} .
$$

Based on the above-mentioned argumentation, one may readily obtain the standardized HFR statistic $S H F R_{s, i}$ and consequently reach the EWMA-type monitoring statistic proposed by Mukherjee et al. (2019), which is given as:

$Z_{s, i}=\max \left(0, \lambda S H F R_{s, i}+(1-\lambda) Z_{s, i-1}\right), i=1,2, \ldots$

where the starting value is equal to zero and $\lambda$ corresponds to the smoothing parameter.

The upper one-sided EWMA nonparametric control chart established by Mukherjee et al. (2019) produces an out-of-control signal if $Z_{s, i}>H$, where $H>0$ denotes the upper control limit.

An extensive numerical experimentation carried out by the same authors, included the utilization of several distributions, such as the Normal distribution, the Exponential distribution, the Gamma distribution, the Log-Logistic distribution, the Laplace distribution and the Log-normal distribution. Based on their results, a variety of interesting comments and instructive remarks are stated in order to shed light on the in-control and out-of-control performance of the proposed EWMA monitoring scheme. It is numerically verified that the new scheme outperforms other traditional nonparametric charts in almost all cases examined, especially for slight and middling changes of the underlying the right-skewed distributions.

Mukherjee et al. (2019) illustrated their proposed nonparametric EWMA schemes by utilizing realworld applications from iron ore mining plant. The measurement of our concern in such data expresses the percentage of silica concentrate that remains as an impurity at the end of the froth flotation process. The outcomes were really encouraging for their EWMA chart.

\section{Conclusions}

Nonparametric EWMA control charts do not require the assumption of any specific probability distribution for the underlying process. In addition, distribution-free control charting is likely to possess the robustness feature of standard nonparametric procedures and consequently there exists less possibility to be affected by outliers, or the presence of skewed or heavy-tailed distributions for the underlying populations. It is evident that the research activity on the topic of nonparametric EWMA control charts is intensively activated and it seems to be strengthened due to their consistent theoretical framework and their appealing practical implementation. Their simplicity and ability to monitor effectively an underlying process even if its distribution is unknown or non-normal, turns them to a very useful statistical tool for improving the quality of a product. Throughout the lines of the present manuscript, a thorough and up-to-date review of relative advances has been taken place, 
International Journal of Mathematical, Engineering and Management Sciences

Vol. 6, No. 3, 708-751, 2021

https://doi.org/10.33889/IJMEMS.2021.6.3.044

while approximately 100 published articles and books in related fields are cited. However, the topic seems to dispose more space for further scientific research. The implementation of additional nonparametric test statistics or their combination with traditional or more purpose-built changepoint models could give birth to new EWMA monitoring schemes. Moreover, the utilization of alternative sampling schemes combined with well-known nonparametric statistics may result in interesting generalizations of the existing charting procedures. Finally, some effort must be done for constructing EWMA nonparametric control charts for joint monitoring plausible changes in location and scale parameters of the underlying distribution process.

\section{Conflicts of Interest}

The authors declare no conflict of interest.

\section{Acknowledgements}

The authors thank two anonymous referees for several helpful comments and suggestions on an earlier version of the manuscript, which resulted in some improvement of the present article.

\section{References}

Abbasi, S.A. (2012). Letter to the editor: A new nonparametric EWMA sign control chart. Expert Systems with Applications: An International Journal, 39(9), 8503.

Abid, M., Nazir, H.Z., Riaz, M., \& Lin, Z. (2016). Use of ranked set sampling in nonparametric control charts. Journal of the Chinese Institute of Engineers, 39(5), 627-636.

Abid, M., Nazir, H.Z., Riaz, M., \& Lin, Z. (2017). An efficient nonparametric EWMA Wilcoxon signed-rank chart for monitoring location. Quality and Reliability Engineering International, 33(3), 669-685.

Abujiya, M.R., \& Muttlak, H. (2004). Quality control chart for the mean using double ranked set sampling. Journal of Applied Statistics, 31(10), 1185-1201.

Alevizakos, V., Chatterjee, K., \& Koukouvinos, C. (2020a). A nonparametric double generally weighted moving average signed-rank control chart for monitoring process location. Quality and Reliability Engineering International, 36(7), 2441-2458. DOI: 10.1002/qre.2706.

Alevizakos, V., Koukouvinos, C., \& Chatterjee, K. (2020b). A nonparametric triple exponentially weighted moving average sign control chart. Quality and Reliability Engineering International, 1-20. DOI: 10.1002/qre.2810.

Ali, S., Abbas, Z., Nazir, H.Z., Riaz, M., Zhang, X., \& Li, Y. (2020). On designing nonparametric EWMA sign chart under ranked set sampling scheme with application to industrial processes. Mathematics, 8(9), 1497.

Al-Omari, A.I., \& Haq, A. (2012). Improved quality control charts for monitoring the process mean, using double ranked set sampling methods. Journal of Applied Statistics, 39(4), 745-763.

Aly, A.A., Hamed, R.M., \& Mahmoud, M.A. (2017). Optimal design of the adaptive exponentially weighted moving average control chart over a range of mean shifts. Communication in Statistics - Simulation and Computation, 46(2), 890-902.

Amin, R.W., \& Searcy, A.J. (1991). A nonparametric exponentially weighted moving average control scheme. Communications in Statistics - Simulation and Computation, 20(4), 1049-1072.

Asghari, S., Gildeh, B.S., Ahmadi, J., \& Borzadaran, G.M. (2018). Sign control chart based on ranked set sampling. Quality Technology \& Quantitative Management, 15(5), 568-588. 
International Journal of Mathematical, Engineering and Management Sciences

Vol. 6, No. 3, 708-751, 2021

https://doi.org/10.33889/IJMEMS.2021.6.3.044

Aslam, M., Azam, M., \& Jun, C.H. (2014). A new exponentially weighted moving average sign chart using repetitive sampling. Journal of Process Control, 24(7), 1149-1153.

Aslam, M., Raza, M.A., Azam, M., Ahmad, L., \& Jun, C.H. (2020). Design of a sign chart using a new EWMA statistic. Communications in Statistics - Theory and Methods, 49(6), 1299-1310.

Balakrishnan, N., Triantafyllou, I.S., \& Koutras, M.V. (2009). Nonparametric control charts based on runs and Wilcoxon-type rank-sum statistics. Journal of Statistical Planning and Inference, 139(9), 31773192.

Balamurali, S., \& Jun, C.H. (2006). Repetitive group sampling procedure for variables inspection. Journal of Applied Statistics, 33(3), 327-338.

Baxley, R.V. Jr. (1990). [Exponentially weighted moving average control schemes: Properties and enhancements]: Discussion. Technometrics, 32(1), 13-16.

Bourke, P.D. (1991). Detecting a shift in fraction nonconforming using run-length control charts with $100 \%$ inspection. Journal of Quality Technology, 23(3), 225-238.

Box, G.E.P., \& Jenkins, G.M. (1976). Time series analysis: forecasting and control. Holden-Day, San Francisco.

Brook, D., \& Evans, D.A. (1972). An approach to the probability distribution of CUSUM run length. Biometrika, 59(3), 539-549.

Brown, R.G., \& Meyer, R.F. (1961). The fundamental theorem of exponential smoothing. Operations Research, 9(5), 673-685.

Capizzi, G., \& Masarotto, G. (2003). An adaptive exponentially weighted moving average control chart. Technometrics, 45(3), 199-207.

Castagliola, P., Tran, K.P., Celano, G., \& Maravelakis, P.E. (2020). The Shewhart sign chart with ties: Performance and alternatives. In Koutras, M.V., \& Triantafyllou, I.S. (eds) Distribution-free Methods for Statistical Process Monitoring and Control, Springer, Cham, pp. 107-136.

Castagliola, P., Tran, K.P., Celano, G., Rakitzis, A.C., \& Maravelakis, P.E. (2019). An EWMA-type sign chart with exact run length properties. Journal of Quality Technology, 51(1), 51-63.

Chakraborti, S., \& Graham, M. (2019a). Nonparametric statistical process control. John Wiley \& Sons, U.S.A.

Chakraborti, S., \& Graham, M.A. (2019b). Nonparametric (distribution-free) control charts: An updated overview and some results. Quality Engineering, 31(4), 523-544.

Chakraborti, S., Van der Laan, P., \& Bakir, S.T. (2001). Nonparametric control charts: An overview and some results. Journal of Quality Technology, 33(3), 304-315.

Cochran, W.G. (2007). Sampling techniques 3rd Edition. Series in Probability and Mathematical Statistics. John Wiley \& Sons.

Dell, T.R., \& Clutter, J.L. (1972). Ranked set sampling theory with order statistics background. Biometrics, 28(2), 545-555.

Frey, J., \& Zhang, Y. (2021). Robust confidence intervals for a proportion using ranked-set sampling. Journal of the Korean Statistical Society. DOI: 10.1007/s42952-020-00103-3.

Gastwirth, J.L. (1965). Percentile modifications of two-sample rank tests. Journal of the American Statistical Association, 60(312), 1127-1141.

Graham, M.A., Chakraborti, S., \& Human, S.W. (2011a). A nonparametric exponentially weighted moving average signed-rank chart for monitoring location. Computational Statistics and Data Analysis, 55(8), 2490-2503. 
International Journal of Mathematical, Engineering and Management Sciences

Vol. 6, No. 3, 708-751, 2021

https://doi.org/10.33889/IJMEMS.2021.6.3.044

Graham, M.A., Chakraborti, S., \& Human, S.W. (2011b). A nonparametric EWMA sign chart for location based on individual measurements. Quality Engineering, 23(3), 227-241.

Graham, M.A., Mukherjee, A., \& Chakraborti, S. (2012). Distribution-free exponentially weighted moving average control charts for monitoring unknown location. Computational Statistics and Data Analysis, 56(8), 2539-2561.

Graham, M.A., Mukherjee, A., \& Chakraborti, S. (2017). Design and implementation issues for a class of distribution-free Phase II EWMA exceedance control charts. International Journal of Production Research, 55(8), 2397-2430.

Hackl, P., \& Ledolter, J. (1991). A control chart based on ranks. Journal of Quality Technology, 23(2), 117124.

Hackl, P., \& Ledolter, J. (1992). A new nonparametric quality control technique. Communications in Statistics - Simulation and Computation, 21(2), 423-443.

Haq, A. (2019). A new nonparametric synthetic EWMA control chart for monitoring process mean. Communications in Statistics - Simulation and Computation, 48(6), 1665-1676.

Haq, A. (2020). A nonparametric EWMA chart with auxiliary information for process mean. Communication in Statistics - Theory and Methods, 49(5), 1232-1247.

Haq, A., Brown, J., Moltchanova, E., \& Al-Omari, A.I. (2015). Effect of measurement error on exponentially weighted moving average control charts under the ranked set sampling schemes. Journal of Statistical Computation and Simulation, 85(6), 1224-1246.

Hawkins, D.M., \& Deng, Q. (2010). A nonparametric change point control chart. Journal of Quality Technology, 42(2), 165-173.

Hettmansperger, T.P. (1995). The ranked-set sample sign test. Journal of Nonparametric Statistics, 4(3), 263-270.

Hogg, R.V., Fisher, D.M., \& Randles, R.H. (1975). A two-sample adaptive distribution-free test. Journal of American Statistical Association, 70(351a), 656-661.

Huang, S., Yang, J., \& Mukherjee, A. (2018). Distribution-free EWMA schemes for simultaneous monitoring of time between events and event magnitude. Computers \& Industrial Engineering, 126, 317-336.

Hunter, J.S. (1986). The exponentially weighted moving average. Journal of Quality Technology, 18(4), 203210.

Hunter, J.S. (1990). [Exponentially weighted moving average control schemes: Properties and enhancements]: Discussion. Technometrics, 32(1), 21-22.

Jurečková, J., \& Kalina, J. (2012). Nonparametric multivariate rank tests and their unbiasedness. Bernoulli, $18(1), 229-251$.

Kim, D.H., \& Kim, Y.C. (1996). Wilcoxon signed rank test using ranked-set sample. Korean Journal of Computational \& Applied Mathematics, 3(2), 235-243.

Koshti, R.D., \& Kamalja, K.K. (2021). Parameter estimation of Cambanis-type bivariate uniform distribution with ranked set sampling. Journal of Applied Statistics, 48(1), 61-83.

Koutras, M.V., \& Triantafyllou, I.S. (2020). Recent advances on univariate distribution-free Shewhart-type control charts. In Koutras, M.V., \& Triantafyllou, I.S. (eds) Distribution-free Methods for Statistical Process Monitoring and Control, Springer, Cham, pp. 1-56.

Kvam, P.H., \& Samaniego, F.J. (1994). Nonparametric maximum likelihood estimation based on ranked set samples. Journal of the American Statistical Association, 89(426), 526-537. 
International Journal of Mathematical, Engineering and Management Sciences

Vol. 6, No. 3, 708-751, 2021

https://doi.org/10.33889/IJMEMS.2021.6.3.044

Lepage, Y. (1971). A combination of Wilcoxon's and Ansari-Bradley's statistics. Biometrika, 58(1), 213217.

Letshedi, T.I., Malela-Majika, J.C., Castagliola, P., \& Shongwe, S.C. (2021). Distribution-free triple EWMA control chart for monitoring the process location using the Wilcoxon rank-sum statistic with fast initial response feature. Quality and Reliability Engineering International. DOI: 10.1002/qre.2842.

Li, S.Y., Tang, L.C., \& Ng, S.H. (2010). Nonparametric CUSUM and EWMA control charts for detecting mean shifts. Journal of Quality Technology, 42(2), 209-226.

Liu, L., Chen, B., Zhang, J., \& Zi, X. (2015). Adaptive Phase II nonparametric EWMA control chart with variable sampling interval. Quality and Reliability Engineering International, 31(1), 15-26.

Liu, L., Peng, Q., Lai, X., \& Deng, Z. (2021). An adaptive nonparametric exponentially weighted moving average control chart with dynamic sampling intervals. Statistical Analysis and Data Mining: The ASA Data Science Journal, 14(1), 74-87.

Liu, L., Zi, X., Zhang, J., \& Wang, Z. (2013). A sequential rank-based nonparametric adaptive EWMA control chart. Communication in Statistics - Simulation and Computation, 42(4), 841-859.

Lu, S.L. (2015). An extended nonparametric exponentially weighted moving average sign control chart. Quality and Reliability Engineering International, 31(1), 3-13.

Lucas, J.M., \& Saccucci, M.S. (1990). Exponentially weighted moving average control schemes: Properties and enhancements. Technometrics, 32(1), 1-12.

MacGregor, J.F., \& Harris, T.J. (1990). [Exponentially weighted moving average control schemes: Properties and enhancements]: Discussion. Technometrics, 32(1), 23-26.

Malela Majika, J.C. (2021). New distribution-free memory-type control charts based on the Wilcoxon ranksum statistic, Quality Technology \& Quantitative Management, 18(2), 135-155.

Malela-Majika, J.C., Adeoti, O.A., \& Rapoo, E. (2018). An EWMA control chart based on the Wilcoxon rank-sum statistic using repetitive sampling. International Journal of Quality \& Reliability Management, 35(3), 711-728.

Mathur, S.K. (2009). A new nonparametric bivariate test for two sample location problem. Statistical Methods and Applications, 18(3), 375.

McCornack, R.L. (1965). Extended tables of the Wilcoxon matched pair signed rank statistic. Journal of American Statistical Association, 60(311), 864-871.

McIntyre, G.A. (1952). A method for unbiased selective sampling, using ranked sets. Australian Journal of Agricultural Research, 3(4), 385-390.

Mehmood, R., Riaz, M., \& Does, R.J.M.M. (2013). Control charts for location based on different sampling schemes. Journal of Applied Statistics, 40(3), 483-494.

Montgomery, D.C. (2009). Introduction to statistical quality control. $6^{\text {th }}$ edition, John Wiley \& Sons, New York.

Mosteller, F., \& Youtz, C. (1961). Tables of the Freeman-Tukey transformations for the binomial and poisson distributions. Biometrika, 48(3/4), 433-440.

Mukherjee, A. (2017). Distribution-free Phase-II exponentially weighted moving average schemes for joint monitoring of location and scale based on subgroup samples. The International Journal of Advanced Manufacturing Technology, 92(1), 101-116.

Mukherjee, A., \& Sen, R. (2015). Comparisons of Shewhart-type rank based control charts for monitoring location parameters of univariate processes. International Journal of Production Research, 53(14), 4414-4445. 
International Journal of Mathematical, Engineering and Management Sciences

Vol. 6, No. 3, 708-751, 2021

https://doi.org/10.33889/IJMEMS.2021.6.3.044

Mukherjee, A., Chong, Z.L., \& Khoo, M.B.C. (2019). Comparisons of some distribution-free CUSUM and EWMA schemes and their applications in monitoring impurity in mining process flotation. Computers \& Industrial Engineering, 137, 106059.

Muttlak, H., \& Al-Sabah, W. (2003). Statistical quality control based on ranked set sampling. Journal of Applied Statistics, 30(9), 1055-1078.

Perdikis, T., Psarakis, S., Castagliola, P., \& Celano, G. (2020a). An EWMA-type chart based on signed ranks with exact run length properties. Journal of Statistical Computation and Simulation, 91(4), 732-751. DOI: $10.1080 / 00949655.2020 .1828415$.

Perdikis, T., Psarakis, S., Castagliola, P., \& Maravelakis, P.E. (2020b). An EWMA signed ranks control chart with reliable run length performances. Quality and Reliability Engineering International, 37(3), 12661284. DOI: $10.1002 /$ qre.2795.

Qiu, P. (2008). Distribution-free multivariate process control based on log-linear modeling. IIE Transactions, 40(7), 664-677.

Qiu, P. (2014). Introduction to statistical process control. CRC Press, Taylor \& Francis Group, New York.

Qiu, P. (2018). Some perspectives on nonparametric statistical process control. Journal of Quality Technology, 50(1), 49-65.

Qiu, P., \& Hawkins, D. (2001). A rank-based multivariate CUSUM procedure. Technometrics, 43(2), 120132.

Qiu, P., \& Li, Z. (2011a). On nonparametric statistical process control of univariate processes. Technometrics, 53(4), 390-405.

Qiu, P., \& Li, Z. (2011b). Distribution-free monitoring of univariate processes. Statistics \& Probability Letters, 81(12), 1833-1840.

Qiu, P., Zi, X., \& Zou, C. (2018). Nonparametric dynamic curve monitoring. Technometrics, 60(3), 386-397.

Qu, L., He, S., Khoo, M.B.C., \& Castagliola, P. (2018). A CUSUM chart for detecting the intensity ratio of negative events. International Journal of Production Research, 56(19), 6553-6567.

Rahali, D., Castagliola, P., Taleb, H., \& Khoo, M.B.C. (2019). Evaluation of Shewhart time-between eventsand-amplitude control charts for several distributions. Quality Engineering, 31(2), 240-254.

Rakitzis, A.C., Castagliola, P., \& Maravelakis, P.E. (2015). A new memory-type monitoring technique for count data. Computers \& Industrial Engineering, 85, 235-247.

Raza, M.A., Nawaz, T., Aslam, M., Bhatti, S.H., \& Sherwani, R.A.K. (2020). A new nonparametric double exponentially weighted moving average control chart. Quality and Reliability Engineering International, 36(1), 68-87.

Reynolds, M.R., Amin, R.W., Arnold, J.C., \& Nachlas, J.A. (1988). $\bar{X}$ charts with variable sampling intervals. Technometrics, 30(2), 181-192.

Riaz, M. (2015). A sensitive non-parametric EWMA control chart. Journal of the Chinese Institute of Engineers, 38(2), 208-219.

Riaz, M., Abid, M., Nazir, H.Z., \& Abbasi, S.A. (2019). An enhanced nonparametric EWMA sign control chart using sequential mechanism. PLoS ONE, 14(11), e0225330.

Riaz, M., Khaliq, Q.U.A., \& Gul, S. (2017). Mixed Tukey EWMA-CUSUM control chart and its applications. Quality Technology and Quantitative Management, 14(4), 378-411.

Roberts, S.W. (1959). Control charts tests based on geometric moving averages. Technometrics, 1(3), 239250. 
International Journal of Mathematical, Engineering and Management Sciences

Vol. 6, No. 3, 708-751, 2021

https://doi.org/10.33889/IJMEMS.2021.6.3.044

Scariano, S.M., \& Calzada, M.E. (2009). The generalized synthetic chart. Sequential Analysis, 28(1), 54-68.

Shafqat, A., Huang, Z., Aslam, M., \& Nawaz, M.S. (2020). A nonparametric repetitive sampling DEWMA control chart based on linear prediction. IEEE Access, 8, 74977-74990.

Shamma, S.E., \& Shamma, A.K. (1992). Development and evaluation of control charts using double exponentially weighted moving averages. International Journal of Quality \& Reliability Management, $9(6), 18-25$.

Sherman, R.E. (1965). Design and evaluation of a repetitive group sampling plan. Technometrics, 7(1), 1121.

Sheu, S.H., \& Lin, T.C. (2003). The generally weighted moving average control chart for detecting small shifts in the process mean. Quality Engineering, 16(2), 209-231.

Song, Z., Mukherjee, A., \& Zhang, J. (2020a). An efficient approach of designing distribution-free exponentially weighted moving average schemes with dynamic fast initial response for joint monitoring of location and scale. Journal of Statistical Computation and Simulation, 90(13), 2329-2353.

Song, Z., Mukherjee, A., Marozzi, M., \& Zhang, J. (2020b). A class of distribution-free exponentially weighted moving average schemes for joint monitoring of location and scale parameters. In Koutras, M.V., \& Triantafyllou, I.S. (eds) Distribution-Free Methods for Statistical Process Monitoring and Control, Springer, Cham, pp. 183-217.

Tang, A., Castagliola, P., Sun, J., \& Hu, X. (2019a). Optimal design of the adaptive EWMA chart for the mean based on median run length and expected median run length. Quality Technology \& Quantitative Management, 16(4), 439-458.

Tang, A., Sun, J., Hu, X., \& Castagliola, P. (2019b). A new nonparametric adaptive EWMA control chart with exact run length properties. Computers \& Industrial Engineering, 130, 404-419.

Triantafyllou, I.S. (2021). Wilcoxon-type rank sum control charts based on progressively censored reference data. Communication in Statistics - Theory and Methods, 50(2), 311-328.

Triantafyllou, I.S., \& Panayiotou, N.I. (2020). A new distribution-free monitoring scheme based on ranks. Communication in Statistics - Simulation and Computation. DOI: 10.1080/ 03610918.2020.1804584.

Wald, A. (1943). Sequential analysis of statistical data: theory, statistical research group, Columbia University.

Wilcoxon, F. (1945). Individual comparisons by ranking methods, Biometrics, 1(6), 80-83.

Woodall, W.H. (2000). Controversies and contradictions in statistical process control (with discussion). Journal of Quality Technology, 32(4), 341-350.

Woodall, W.H., \& Montgomery, D.C. (1999). Research issues and ideas in statistical process control. Journal of Quality Technology, 31(4), 376-386.

Wu, S., Castagliola, P., \& Celano, G. (2020). A distribution-free EWMA control chart for monitoring timebetween-events-and-amplitude data. Journal of Applied Statistics, 48(3), 434-454. DOI: $10.1080 / 02664763.2020 .1729347$.

Wu, Z., \& Spedding, T.A. (2000). A synthetic control chart for detecting small shifts in the process mean. Journal of Quality Technology, 32(1), 32-38.

Wu, Z., Jiao, J., \& He, Z. (2009). A control scheme for monitoring the frequency and magnitude of an event. International Journal of Production Research, 47(11), 2887-2902.

Xue, L., \& Qiu, P. (2020). A nonparametric CUSUM chart for monitoring multivariate serially correlated processes. Journal of Quality Technology. DOI: 10.1080/00224065.2020. 1778430. 
International Journal of Mathematical, Engineering and Management Sciences

Vol. 6, No. 3, 708-751, 2021

https://doi.org/10.33889/IJMEMS.2021.6.3.044

Yang, S.F., Lin, J.S., \& Cheng, S.W. (2011). A new nonparametric EWMA sign control chart. Expert Systems with Applications, 38(5), 6239-6243.

You, L., \& Qiu, P. (2020). A nonparametric control chart for dynamic disease risk monitoring. In Koutras, M.V., \& Triantafyllou, I.S. (eds) Distribution-free Methods for Statistical Process Monitoring and Control, Springer, pp. 243-257.

Zou, C., \& Tsung, F. (2010). Likelihood ratio-based distribution-free EWMA control charts. Journal of Quality Technology, 42(2), 174-196. 